MS-TPI-93-09

\title{
Orbifold Compactifications with Continuous Wilson Lines
}

\author{
Thomas Mohaupt政 \\ Institut für Theoretische Physik I, Universität Münster \\ Wilhelm-Klemm-Str. 9, D-48149 Münster, Germany \\ October 27, 1993门
}

\begin{abstract}
:
We identify the untwisted moduli of heterotic orbifold compactifications for the case, when the gauge twist is realized by a rotation. The Wilson lines are found to have both continuous and discrete parts. For the case of the standard $\mathbf{Z}_{3}$ orbifold we classify all possibilities of breaking the gauge group $E(6) \otimes S U(3)$ by nine of the eighteen Wilson moduli and by additional discrete Wilson lines.
\end{abstract}

\footnotetext{
*New address from 1 november 1993: DESY-IfH Zeuthen, Platanenallee 6, D-15738 Zeuthen. (Postal address: P.O. Box, D-15735 Zeuthen. E-mail: mohaupt at ifh.de.)

${ }^{\dagger}$ Corrected: November 8, 1993.
} 


\section{Introduction}

The compactification of the heterotic string theory [1] from ten to four dimensions can be realized in a lot of different ways [2]. A generic feature of all these schemes is the appearence of contiuous deformation parameters, called moduli [3, 4. The moduli spaces of toroidal [8, 9] and orbifold compactifications [5, 6, 7] have been studied extensively during the last years. This is due to the fact that these models are exactly solvable and lead, at least for some orbifolds, to semirealistic physics [10, 11]. Besides being of some phenomenological interest, they are a good laboratory for ideas about string theory that may later be generalized to more general compactifications or internal conformal field theories.

Perhaps the most prominent point of interest is target space modular invariance, a symmetry of the moduli space, which is a genuine stringy phenomenon 12, 13, 1 . Since this modular group contains inversions of the radius of the internal manifold, it indicates the existence of a fundamental length scale. On the other hand, demanding the absence of modular anomalies puts severe restrictions on the effective Lagrangean describing the low (compared to Planck scale) energy regime [14, 15].

Another point of interest is the dependence of the gauge group on the moduli [16, 17]. This is not only useful for identifying models with standard model or GUT gauge groups, but also linked with target space modular invariance, because points of maximally extended symmetry are often fixed points of the modular group [18. It has been argued that both the continuous, finite dimensional gauge group and the discrete modular group are remnants of the full, (spontanously broken) infinite dimensional, continuous string symmetry group [19].

It is therefore surprising that a big part of the orbifold moduli space has not yet been explored systematically: The moduli associated with continuous Wilson lines. These moduli are known to exist, when the gauge twist is not realized by a shift, but by a rotation [20]. In this paper we will show that it is possible in the case of $\mathbf{Z}_{N}$ orbifolds to determine all (untwisted moduli including the Wilson moduli. Our approach will be similar to that used in [20], namely we will analyze the constraint that the twist must be an automorphism of the momentum lattice of the underlying toroidal model. In [20] this was applied to symmetric orbifolds with gauge twist realized by a shift and to general asymmetric orbifolds, whereas the case which we will study (called symmetric orbifolds with non abelian embedding in [20] ) was only mentioned but not treated explicitly. Note also that in [20] no formula was derived for the number of moduli. Since this has been shown to be possible more recently in 21] for bosonic orbifolds, we will try to do the same in the heterotic case. Our second task will be to characterize the moduli dependence of the gauge group. This will be done using the results for toroidal models derived in [17].

The organisation of this paper is as follows: In the next section we will recall some background material. Then the constraints resulting from demanding consistency between a given twist of the momentum lattice and the background fields will be worked out. By analyzing the constraints we will learn, which parts of the Wilson lines can be varied continuously and which are discrete. As a byproduct we will show that discrete ("quantized") background fields reflect the remaining freedom of selecting different twists of the momentum lattice, when a target space twist 3 has already been chosen. Then we will define the gauge twist in terms of the target space twist and derive formulas for the number of moduli. The general results will then be illustrated taking the standard $\mathbf{Z}_{3}$ Orbifold as an example. We will classify all possibilities of breaking the gauge group $E(6) \otimes S U(3)$ by nine of the eighteen Wilson moduli and by additional discrete Wilson lines.

\footnotetext{
${ }^{1}$ The so called twisted moduli, which are associated with the blowing up of orbifold singularities will not be studied here.

${ }^{2}$ Such orbifolds are called asymmetric by other authors, because they generically have $(0,2)$ world sheet supersymmetry only.

${ }^{3}$ The terminology will be explained in the next section.
} 


\section{Background material}

Toroidal orbifold models are constructed by modding out a discrete symmetry of a toroidal compactification of the ten dimensional heterotic string theory. We must therefore recall some elements of the Narain model [8, 9].

\subsection{Toroidal compactifications}

The Narain model desribes strings moving in a space-time $\mathbf{M}^{10-d} \times \mathbf{T}^{d}$, where $\mathbf{M}^{10-d}$ is a $(10-d)$-dimensional Minkowski space and $\mathbf{T}^{d}$ is a $d$-dimensional torus, defined by

$$
\mathbf{T}^{d}=\mathbf{R}^{d} / \Lambda,
$$

where $\Lambda$ is a $d$-dimensional lattice. In the following we will need a lattice basis $\mathbf{e}_{i}$ of $\Lambda$,

$$
\Lambda=\left\langle\mathbf{e}_{i} \mid i=1, \ldots, d\right\rangle_{\mathbf{Z}}:=\left\{m^{i} \mathbf{e}_{i} \mid m^{i} \in \mathbf{Z}\right\} .
$$

Inequivalent choices of $\Lambda$ can be parametrized by the lattice metric

$$
G_{i j}:=\mathbf{e}_{i} \cdot \mathbf{e}_{j}
$$

They correspond to different background metrics in the $d$ internal directions. But there are further continuous deformations: One can also introduce a constant antisymmetric background field $B_{i j}$, sometimes called axion and a constant background gauge field of the ten-dimensional gauge group, which can be paramatrized by six vectors $\mathbf{A}_{i} \in \mathbf{R}^{16}$, called Wilson lines [9]. A basis of the Hilbert space of the model is provided by acting with transverse oszillators on the ground states

$$
\left|\left(\mathbf{K}, \mathbf{k}_{L} ; \mathbf{k}_{R}\right)\right\rangle
$$

of the different charge/winding/momentum sectors. The vectors $\left(\mathbf{K}, \mathbf{k}_{L} ; \mathbf{k}_{R}\right)$ are constrained to form an even lattice $\Gamma_{16+d ; d}$, which is selfdual with respect to an indefinite bilinear form with signature $(+)^{16+d}(-)^{d}[8]$. By varying the background fields one can deform all (physically inequivalent) lattices of this type into each other. The effect of such deformations can be made transparent by chosing a special basis of $\Gamma_{16+d ; d}$ [16. This basis is constructed out of a basis $\mathbf{e}_{i}$ of the compactification lattice $\Lambda$, a basis $\mathbf{e}^{* i}$ of its dual $\Lambda^{*}$ and a basis $\mathbf{e}_{A}$ of the even selfdual euclidean lattice $\Gamma_{16}$, which contains the gauge quantum numbers of the corresponding ten-dimensional theory:

$$
\begin{gathered}
\mathbf{k}^{i}=\left(0, \frac{1}{2} \mathbf{e}^{* i} ; \frac{1}{2} \mathbf{e}^{* i}\right), \\
\overline{\mathbf{k}}_{i}=\left(\mathbf{A}_{i}, 2 \mathbf{e}_{i}+D_{i j} \frac{1}{2} \mathbf{e}^{* j} ; D_{i j} \frac{1}{2} \mathbf{e}^{* j}\right), \\
\mathbf{l}_{A}=\left(\mathbf{e}_{A},-\left(\mathbf{e}_{A} \cdot \mathbf{A}_{k}\right) \frac{1}{2} \mathbf{e}^{* k} ;-\left(\mathbf{e}_{A} \cdot \mathbf{A}_{k}\right) \frac{1}{2} \mathbf{e}^{* k}\right),
\end{gathered}
$$

where

$$
D_{i j}=2\left(B_{i j}-G_{i j}-\frac{1}{4}\left(\mathbf{A}_{i} \cdot \mathbf{A}_{j}\right)\right) .
$$

and $i, j, k=1, \ldots, d, A=1, \ldots, 16$. For all values of the background fields the only nonvanishing (pseudo-euclidean) scalar products are

$$
\mathbf{k}^{i} \cdot \overline{\mathbf{k}}_{j}=\delta_{j}^{i} \text { and } \mathbf{l}_{A} \cdot \mathbf{l}_{B}=\mathbf{e}_{A} \cdot \mathbf{e}_{B}=: G_{A B} .
$$

This basis is also useful for the definition of the twist.

\footnotetext{
${ }^{4}$ From the four-dimensional point of view the components of all thesese vectors are charge-like quantum numbers.
} 


\subsection{Orbifold compactifications}

The most general way to twist the Narain model is to divide out some discrete group of automorphisms of the Hilbert space [7, 18]. This is usually done by specifying automorphisms of the momentum lattice $\Gamma=\Gamma_{16+d ; d}$ of the torus model [7]. We will restrict our attention to

- cyclic groups of automorphims, which are generated by a single twist $\Theta$ of finite order, $\Theta^{N}=1$,

- and to models, which admit an geometric interpretation as compactification on a toroidal orbifold [5, 6].

To fulfill the second condition, the lattice twist $\Theta$ must come from a target space twist $\theta \in O(d)$, $\theta^{N}=\mathbf{1}$, which defines the target space $\mathbf{O}^{d}$ of the $d$ internal coordinates,

$$
\mathbf{O}^{d}=\mathbf{T}^{d} /\langle\theta\rangle \text {. }
$$

This is a well defined toroidal orbifold, provided $\theta$ is a lattice automorphism of $\Lambda$.

Since the states $\left|\left(\mathbf{K}, \mathbf{k}_{L} ; \mathbf{k}_{R}\right)\right\rangle$ are created by vertex operators

$$
\exp \left(K_{A} X_{L}^{A}(z)+k_{L, i} X_{L}^{i}(z)-k_{R, i} X_{R}^{i}(\bar{z})\right)
$$

the choice of a target space twist $\theta$ acting on $X^{i}(z, \bar{z})=X_{L}^{i}(z)+X_{R}^{i}(\bar{z})$ does not only specify the action of the twist on the oszillators, but also fixes the action of $\Theta$ on the $d+d$ quantum numbers $k_{L, i}, k_{R, i}$ of a state. We are, however, free to define the action of $\Theta$ on the 16 components $K_{A}$ by specifying a gauge twist. It is necessary to choose a gauge twist different from the identity, because otherwise a rank sixteen factor of the gauge group of the toroidal model remains unbroken. In general the gauge twist can be an element of the sixteen dimensional Euclidean group. The most studied possibility is that of a pure shift (translation). This is technically the simplest case, but it has two drawbacks [20]:

- The modding out by shifts leaves at least one $U(1)$ gauge group per direction unbroken. Therefore the rank of the gauge group cannot be reduced. In the case of heterotic orbifolds the rank is at least 16, which is much bigger than the rank of the gauge group of the standard model.

- Whereas some components of the metric and axionic background field can still be varied continuously, the Wilson lines are only allowed to take discrete values. Combined with point 1 this suggests that one is only constructing special points of extended symmetry in a bigger moduli space.

It is well known that if the gauge twist is a rotation (or contains a rotation) the rank of the gauge group is generically reduced and that some components of the Wilson lines can still be varied continuously [20]. This has, however, not been studied systematically enough to answer questions like these:

1. What are the most general background fields compatible with a given twist?

2. What is the dimension of the moduli space? Which components of the Wilson lines are moduli and which are discrete?

3. How does the gauge group depend on the moduli? What patterns of symmetry breaking do appear?

These questions will be attacked in the following. For simplicity the gauge twist will be a pure rotation, $\theta^{\prime} \in O(16)$, which must be an automorphism of $\Gamma_{16}$ in order to be compatible with the toric boundary conditions of the sixteen extra left moving coordinates $X_{L}^{A}$. Sometimes the gauge twist is defined in terms of the target space twist. This imitates the embedding of the spin connection into the gauge connection in Calabi-Yau compactifications [6]. We will 
postpone the specification of the gauge twist and try to learn first to what extent it is restricted by simply demanding that $\Theta$ is an lattice automorphism of $\Gamma_{16+d ; d}$. It will only be restricted to have the same order as the target space twist, $\theta^{\prime N}=1$, so that $\Theta$ has also order N.

In order to derive the consistency conditions between the twist $\Theta$ and the background fields, it is convenient to introduce the following matrices, which characterize the action of the twist on the bases $\mathbf{e}_{i}, \mathbf{e}^{* i}$ and $\mathbf{e}_{A}, i=1, \ldots, d, A=1, \ldots, 16$ of the lattices $\Lambda, \Lambda^{*}$ and $\Gamma_{16}$ :

$$
\Theta:\left\{\begin{array}{r}
\mathbf{e}_{i} \rightarrow \theta \mathbf{e}_{i}=\theta_{i}{ }^{j} \mathbf{e}_{j}, \\
\mathbf{e}^{* i} \rightarrow \theta \mathbf{e}^{* i}=\theta_{j}^{i} \mathbf{e}^{* j}, \\
\mathbf{e}_{A} \rightarrow \theta^{\prime} \mathbf{e}_{A}=\theta_{A}{ }^{B} \mathbf{e}_{B} .
\end{array}\right.
$$

We can now start to analyze the basic condition

$$
\Theta \in \operatorname{Aut}\left(\Gamma_{16+d ; d}\right)
$$

in order to identify the admissible continuous and discrete deformations of $\Gamma_{16+d ; d}$.

\section{Derivation of the consistency equations}

The action of the twist on the momentum lattice is given by (12). $\Theta$ is an automorphism of $\Gamma_{16+d ; d}$ if and only if the image of each basis vector is again a lattice vector, that is an integer linear combination of basis vectors. This will be worked out in the following.

\subsection{The basis vectors $\mathrm{k}^{i}$}

The first set of conditions is

$$
\Theta \mathbf{k}^{i}=\left(0, \frac{1}{2} \theta_{j}^{i} \mathbf{e}^{* j} ; \frac{1}{2} \theta_{j}^{i} \mathbf{e}^{* j}\right) \stackrel{!}{=} M_{j}^{i} \mathbf{k}^{j}+M^{i j} \overline{\mathbf{k}}_{j}+M^{i A} \mathbf{l}_{A},
$$

with $M_{j}^{i}, M^{i j}, M^{i A} \in \mathbf{Z}$. Comparing with (5) - (7) immediately gives

$$
M_{j}^{i}=\theta_{j}^{i}, \quad M^{i j}=0, \quad M^{i A}=0 .
$$

The first of these equations means that $\theta$ must be an automorphism (of $\Lambda^{*}$ and therefore) of $\Lambda$. This condition is trivial from the geometrical point of view, since the action of $\theta$ must result in a well defined orbifold $\mathbf{O}^{6}=\mathbf{T}^{6} /\langle\theta\rangle$. It is however interesting that it follows automatically from the basic condition (13). The other two equations simply tell us that certain elements of the matrix of $\Theta$ (w.r.t. the basis (5) - (7) ) must vanish.

\subsection{The basis vectors $\overline{\mathbf{k}}_{i}$}

The second set of conditions is less simple:

$$
\Theta \overline{\mathbf{k}}_{i}=\left(\theta^{\prime} \mathbf{A}_{i}, 2 \theta_{i}^{j} \mathbf{e}_{j}+\frac{1}{2} D_{i j} \theta_{k}^{j} \mathbf{e}^{* k} ; \frac{1}{2} D_{i j} \theta_{k}^{j} \mathbf{e}^{* k}\right) \stackrel{!}{=} M_{i j} \mathbf{k}^{j}+M_{i}^{j} \overline{\mathbf{k}}_{j}+M_{i}{ }^{A} \mathbf{l}_{A},
$$

with $M_{i j}, M_{i}{ }^{j}, M_{i}{ }^{A} \in \mathbf{Z}$. We can now use again (5) - (7). The resulting equation can be seperated into three equations, the first one for the first sixteen components, the second for the next six components and the third for the last six components. Taking the difference between the second and third equation yields

$$
2 \theta_{i}^{j} \mathbf{e}_{j}=2 M_{i}^{j} \mathbf{e}_{j}
$$

Since the $\mathbf{e}_{i}$ are linearly independent, we get

$$
\theta_{i}^{j}=M_{i}^{j}
$$


which again states that $\theta \in \operatorname{Aut}(\Lambda)$. Using this, the first and third equation are

$$
\begin{gathered}
\theta^{\prime} \mathbf{A}_{i}=\theta_{i}{ }^{j} \mathbf{A}_{j}+M_{i}{ }^{A} \mathbf{e}_{A}, \\
D_{i j} \theta_{k}^{j}-\theta_{i}^{j} D_{j k}=M_{i k}-M_{i}{ }^{A}\left(\mathbf{e}_{A} \cdot \mathbf{A}_{k}\right) .
\end{gathered}
$$

Equation (19) implies that, up to a lattice vector of $\Gamma_{16}$, the Wilson lines must transform under the gauge twist $\theta^{\prime}$ like the directions to which they are assigned to transform under the target space twist $\theta$. This is a generalization of the usual construction of the gauge twist by embedding the target space translation group into the gauge group [6], where no lattice vector is allowed $\left(M_{i}{ }^{A}=0\right)$.

Equation (20) shows that the D-matrix (8) must commute with the twist $\theta$, except for the right hand side. It resembles the equations which give the number of moduli in bosonic orbifolds and heterotic orbifolds without Wilson lines [21]. Both equations will be analyzed in the next section.

We close this subsection by giving formulas for the matrix elements $M_{i}{ }^{A}, M_{i k}$ of the momentum lattice twist $\Theta$. This can be done by introducing a matrix $G^{A B}$, which is the inverse of the lattice metric

$$
\mathbf{e}_{A} \cdot \mathbf{e}_{B}=G_{A B}
$$

of $\Gamma_{16}$. The result is:

$$
\begin{gathered}
M_{i}{ }^{A}=G^{A B}\left(\left(\theta^{\prime} \mathbf{A}_{i}-\theta_{i}{ }^{j} \mathbf{A}_{j}\right) \cdot \mathbf{e}_{B}\right) . \\
M_{i k}=D_{i j} \theta_{k}^{j}-\theta_{i}^{j} D_{j k}+\left(\theta^{\prime} \mathbf{A}_{i}-\theta_{i}{ }^{j} \mathbf{A}_{j}\right) \cdot \mathbf{A}_{k} .
\end{gathered}
$$

\subsection{The basis vectors $l_{A}$}

The third set of consistency conditions is

$$
\Theta \mathbf{l}_{A}=\left(\theta^{\prime} \mathbf{e}_{A},-\frac{1}{2}\left(\mathbf{e}_{A} \cdot \mathbf{A}_{i}\right) \theta_{j}^{i} \mathbf{e}^{* j} ;-\frac{1}{2}\left(\mathbf{e}_{A} \cdot \mathbf{A}_{i}\right) \theta_{j}^{i} \mathbf{e}^{* j}\right) \stackrel{!}{=} M_{A i} \mathbf{k}^{i}+M_{A}^{i} \overline{\mathbf{k}}_{i}+M_{A}^{B} \mathbf{l}_{B},
$$

with $M_{A i}, M_{A}^{i}, M_{A}^{B} \in \mathbf{Z}$. Applying the same procedure as in the last subsection one derives

$$
M_{A}^{i}=0 .
$$

Substituting this in the equation for the first sixteen components gives

$$
\theta^{\prime} \mathbf{e}_{A}=M_{A}^{B} \mathbf{e}_{B},
$$

which means that the gauge twist is a lattice automorphism of $\Gamma_{16}$. Again we have rederived a condition which is sometimes stated independently from the basic condition (13). Using (5) we have

$$
M_{A}^{B}=\theta_{A}^{B} .
$$

Using the previous results, the equation for the last six components can be brought into the form

$$
\mathbf{e}_{A} \cdot\left(\theta^{\prime-1} \mathbf{A}_{j}-\mathbf{A}_{i} \theta_{j}^{i}\right)=M_{A j} .
$$

This means that $\left(\theta^{\prime-1} \mathbf{A}_{j}-\mathbf{A}_{i} \theta^{i}\right)$ must be in $\Gamma_{16}$, because this lattice is selfdual. It is however no new condition, since it is equivalent to (19). (This is easily seen using $\theta^{N}=\mathbf{1}, \theta^{\prime N}=\mathbf{1}$.)

Thus we achieved two results: The first is that the basic condition $\Theta \in \operatorname{Aut}\left(\Gamma_{16+d ; d}\right)$ is equivalent to the equations (19), 20) and $\theta, \theta^{\prime}$ being automorphisms of $\Lambda, \Gamma_{16}$. The second is an explicit matrix representation of $\Theta$ w.r.t. the basis (5) - (7) in terms of the matrices of $\theta, \theta^{\prime}$ and the background fields. It is given by equations (15), (18), (22), (23), (25), (27) and (28). 


\section{Analysis of the consistency equations}

In this section the two consistency equations (19) and (20) will be analyzed. One useful tool for dealing with such equations is to refer them not to a lattice basis but to an orthonormal basis [21. This is simple linear algebra, but can easily cause confusion. Therefore we will collect some useful formulas and introduce notation first.

\subsection{Lattice basis and orthonormal basis}

In equations (19) and (20) the target space twist $\theta$, which is an orthogonal map on the real vector space $\langle\Lambda\rangle_{\mathbf{R}} \simeq \mathbf{R}^{d}$, appears via its matrices w.r.t. to the lattice bases of $\Lambda$ and $\Lambda^{*}$ :

$$
M_{\left\{\mathbf{e}_{i}\right\}}(\theta)=\vartheta=\left(\theta_{i}^{j}\right), \quad M_{\left\{\mathbf{e}^{* i}\right\}}(\theta)=\vartheta^{*}=\left(\theta^{i}{ }_{j}\right)
$$

Since a generic lattice is neither orthogonal nor selfdual, the matrix $\vartheta$ is in general not orthogonal and not equal to $\vartheta^{*}$. But the orthogonality of the map $\theta$ is reflected in the relation

$$
\vartheta^{*}=\vartheta^{T,-1} .
$$

If we refer $\theta$ to an orthonormal basis $\mathbf{e}_{\mu}$,

$$
M_{\left\{\mathbf{e}_{\mu}\right\}}(\theta)=\bar{\vartheta}=\left(\theta_{\mu}^{\nu}\right)
$$

then the matrix $\bar{\vartheta}$ is orthogonal and selfdual (w.r.t. our ${ }^{*}{ }_{-}$operation):

$$
\bar{\vartheta} \bar{\vartheta}^{T}=\mathbf{1}, \quad \bar{\vartheta}=\bar{\vartheta}^{*}
$$

This will be used to simplify equations (19), (20) later.

The two bases are connected by an invertible matrix $\mathbf{T}$, defined by

$$
\mathbf{T}=\left(T_{i}^{\mu}\right), \quad \mathbf{e}_{i}=T_{i}^{\mu} \mathbf{e}_{\mu}
$$

The inverse matrix is denoted by $\mathbf{T}^{-1}=\left(T_{\mu}^{i}\right)$. The dual bases are connected by

$$
\mathbf{e}^{* i}=\mathbf{e}^{* \mu} T_{\mu}^{i}
$$

and the relation between the various matrices represending $\theta$ is

$$
\bar{\vartheta}=\mathbf{T}^{-1} \vartheta \mathbf{T}=\mathbf{T}^{T} \vartheta^{*} \mathbf{T}^{T,-1}
$$

When dealing with equations (19) and (20) we must also transform the vectors $\mathbf{A}_{i}$ and

$$
\mathbf{v}_{i}:=M_{i}{ }^{A} \mathbf{e}_{A}
$$

by

$$
\mathbf{A}_{\mu}=T_{\mu}^{i} \mathbf{A}_{i}, \quad \mathbf{v}_{\mu}=T_{\mu}^{i} \mathbf{v}_{i} .
$$

This must not be confused with a basis transformation since $\mathbf{A}_{i}$ and $\mathbf{v}_{i}$ are not vectors in $\langle\Lambda\rangle_{\mathbf{R}}$, but in a different, sixteen dimensional vector space $\left\langle\Gamma_{16}\right\rangle_{\mathbf{R}} \simeq \mathbf{R}^{16}$. We are simply using the invertible matrix $\mathbf{T}$ to rearrange them into new linear combinations 5 . Note also that the vectors $\mathbf{v}_{i}$ by definition are lattice vectors of $\Gamma_{16}$ whereas the $\mathbf{v}_{\mu}$ generically are not. Likewise the matrices $\vartheta, \vartheta^{*}$ are integer matrices, because they describe a lattice automorphism w.r.t. a lattice basis, whereas $\bar{\vartheta}$ will in general be no integer matrix.

Some final technical remarks: In the following component and matrix notation as defined here will be freely interchanged. Indices referring to the orthonormal basis will be written lowercase, if no misinterpretation is possible (for example $\theta_{\mu \nu}:=\theta_{\mu}^{\nu}=\theta_{\nu}^{\mu}$ ).

\footnotetext{
${ }^{5}$ In the case of Wilson lines this can be interpreted as another decomposition of the gauge connection: $\mathbf{A}=\mathbf{A}_{i} d x^{i}=\mathbf{A}_{\mu} d x^{\mu}$.
} 


\subsection{Continuous and discrete Wilson lines}

The next step is to show that equation (19) decides which components of the Wilson lines can be varied continuously in our orbifold model and which components are restricted to a discrete set of values. Referring to an orthonormal basis, we get:

$$
\theta^{\prime} \mathbf{A}_{\mu}=\theta_{\mu \nu} \mathbf{A}_{\nu}+\mathbf{v}_{\mu}
$$

In order to simplify this equation we decompose each Wilson line in a part $\widetilde{\mathbf{A}}_{\mu}$ which is invariant under the gauge twist and its complement $\widehat{\mathbf{A}}_{\mu}$. This decomposition is both orthogonal and direct (i.e. unique):

$$
\mathbf{A}_{\mu}=\widehat{\mathbf{A}}_{\mu}+\widetilde{\mathbf{A}}_{\mu}, \quad \theta^{\prime} \widetilde{\mathbf{A}}_{\mu}=\widetilde{\mathbf{A}}_{\mu} .
$$

Doing the same with the inhomogeneous term $\mathbf{v}_{\mu}$ in (38) we get the two equations

$$
\theta^{\prime} \widetilde{\mathbf{A}}_{\mu}=\widetilde{\mathbf{A}}_{\mu}=\theta_{\mu \nu} \widetilde{\mathbf{A}}_{\nu}+\widetilde{\mathbf{v}}_{\mu}, \quad \theta^{\prime} \widehat{\mathbf{A}}_{\mu}=\theta_{\mu \nu} \widehat{\mathbf{A}}_{\nu}+\widehat{\mathbf{v}}_{\mu} .
$$

These equations can be decomposed further, if we seperate such directions on $\mathbf{T}^{6}$ which are invariant under the target space twist $\theta$ from those which are non-invariant. This is however (in general) not possible w.r.t. the lattice basis $\mathbf{e}_{i}$ of $\Lambda$. But there always exists an orthonormal basis $\mathbf{e}_{\mu}$ such that the matrix of the twist is block-diagonal

$$
\left(\theta_{\mu \nu}\right)=\left(\begin{array}{cc}
\theta_{a b} & \mathbf{0} \\
\mathbf{0} & \delta_{m n}
\end{array}\right),
$$

where $a, b=1, \ldots, d-M, m, n=d-M+1, \ldots, d . M$ is the dimension of the invariant subspace of $\theta$ and the matrix $\left(\theta_{a b}\right)$ does not have the eigenvalue 1. By (41) the transformed equations (40) decouple into four equations. There are four different kinds of Wilson lines 8 , each subject to a different constraint:

1. Non-invariant Wilson lines, which are assigned to a non-invarant direction in target space:

$$
\theta^{\prime} \widehat{\mathbf{A}}_{a}-\theta_{a b} \widehat{\mathbf{A}}_{b}=\widehat{\mathbf{v}}_{a} .
$$

This is an inhomogeneous linear equation for the $\widehat{\mathbf{A}}_{a}$. Since the corresponding homogeneous equation has nontrivial solutions 9 , these Wilson lines can be varied continuously. To calculate the number of moduli we must take into account that they are further constrained by equation (20). This will be done in the next section.

2. Invariant Wilson lines assigned to non-invariant directions in target space:

$$
\theta^{\prime} \widetilde{\mathbf{A}}_{a}-\theta_{a b} \widetilde{\mathbf{A}}_{b}=\widetilde{\mathbf{A}}_{a}-\theta_{a b} \widetilde{\mathbf{A}}_{b}=\widetilde{\mathbf{v}}_{a} .
$$

Since the matrix $\left(\theta_{a b}\right)$ has no eigenvalue 1 , we could argue that the the corresponding homogeneous equation has only the trivial solution and the solution of the inhomogeneous equation is unique. But this argument does not apply, when the $\widetilde{\mathbf{A}}_{a}$ are not linear independent. Fortunately we can modify a trick from [20], which allows us to solve (43) for the Wilson lines. We can iteratively apply $\theta^{\prime}$ to equation (43) and use the fact, that $\widetilde{\mathbf{A}}_{a}$ and $\widetilde{\mathbf{v}}_{a}$ are invariant:

$$
\widetilde{\mathbf{A}}_{a}=\theta^{\prime} \widetilde{\mathbf{A}}_{a}=\theta_{a b} \widetilde{\mathbf{A}}_{b}+\widetilde{\mathbf{v}}_{a}
$$

\footnotetext{
${ }^{6}$ Such a decomposition is of cause also possible for the $\mathbf{A}_{i}$. We are, however, preparing a second decomposition, which in general forces us to switch to the $\mathbf{A}_{\mu}$.

${ }^{7}$ The author thanks D. Jungnickel for pointing this out to him. See also [22] for implications on the classification of orbifolds.

${ }^{8}$ Although $\widehat{\mathbf{A}}_{a}$ and $\widetilde{\mathbf{A}}_{a}$ are strictly speaking different projections of one Wilson line $\mathbf{A}_{a}$, we will loosely refer to them as "different Wilson lines", because they decouple and behave quite differently, as shown in the text.

${ }^{9}$ We could for example simply define the gauge twist by $\theta^{\prime} \widehat{\mathbf{A}}_{a}=\theta_{a b} \widehat{\mathbf{A}}_{b}$.
} 


$$
\begin{gathered}
\widetilde{\mathbf{A}}_{a}=\theta^{\prime 2} \widetilde{\mathbf{A}}_{a}=\theta_{a b} \theta^{\prime} \widetilde{\mathbf{A}}_{b}+\widetilde{\mathbf{v}}_{a}= \\
=\theta_{a b}\left(\theta_{b c} \widetilde{\mathbf{A}}_{c}+\widetilde{\mathbf{v}}_{b}\right)+\widetilde{\mathbf{v}}_{a}=\theta_{a b} \theta_{b c} \widetilde{\mathbf{A}}_{c}+\widetilde{\mathbf{v}}_{a}+\theta_{a b} \mathbf{v}_{b}
\end{gathered}
$$

and so on. Since $\theta^{N}=\mathbf{1}$, the sum of the first $\mathrm{N}$ powers of $\left(\theta_{a b}\right)$ projects onto the invariant subspace. But $\left(\theta_{a b}\right)$ has no eigenvalue 1, implying

$$
\theta_{a b}+\theta_{a c} \theta_{c b}+\cdots \underbrace{\theta_{a c} \cdots \theta_{d b}}_{\mathrm{N} \text { factors }}=\mathbf{0} .
$$

Adding the first $\mathrm{N}$ equations produced by iteration and using this identity, we arrive at an explicit expression for $\widetilde{\mathbf{A}}_{a}$ in terms of $\widetilde{\mathbf{v}}_{a}$ :

$$
\widetilde{\mathbf{A}}_{a}=\frac{N-1}{N} \widetilde{\mathbf{v}}_{a}+\frac{N-2}{N} \theta_{a b} \widetilde{\mathbf{v}}_{b}+\cdots+\frac{1}{N} \underbrace{\theta_{a c} \cdots \theta_{d b}}_{\text {N-2 factors }} \widetilde{\mathbf{v}}_{b} .
$$

Therefore the Wilson lines $\widetilde{\mathbf{A}}_{a}$ can only take discrete values 10 .

3. Non-invariant Wilson lines assigned to invariant directions in target space:

$$
\theta^{\prime} \widehat{\mathbf{A}}_{m}-\widehat{\mathbf{A}}_{m}=\widehat{\mathbf{v}}_{m} .
$$

The Wilson lines $\widehat{\mathbf{A}}_{m}$ are by definition orthogonal to the invariant subspace of the gauge twist $\theta^{\prime}$. The homogeneous equation has only the trivial solution, the Wilson lines are discret $[$. A formula similar to (47) can be derived by the iterative trick:

$$
\widehat{\mathbf{A}}_{m}=-\frac{N-1}{N} \widehat{\mathbf{v}}_{m}-\cdots-\frac{1}{N} \theta^{\prime N-2} \widehat{\mathbf{v}}_{m} .
$$

4. Invariant Wilson lines assigned to invariant directions in target space:

$$
\theta^{\prime} \widetilde{\mathbf{A}}_{m}-\widetilde{\mathbf{A}}_{m}=0=\widetilde{\mathbf{v}}_{m} .
$$

This equation is identically fulfilled for the Wilson lines $\widetilde{\mathbf{A}}_{m}$, which can be varied continuously.

Thus we have shown that equation (19) can be used to disentangle the discrete and continouus parts of the Wilson lines. The resulting picture is simple and symmetric: (non) invariant Wilson lines corresponding to (non) invariant directions are continuous, whereas invariant (noninvariant) Wilson lines corresponding ton non-invariant (invariant) directions are discrete.

Our equations can be used to analyze the role of discrete parameters further. Obviously there are three kinds of discrete parameters connected with the Wilson lines: Beside the discrete Wilson lines $\widetilde{\mathbf{A}}_{a}$ and $\widehat{\mathbf{A}}_{m}$ we must specify a particular solution $\widehat{\mathbf{A}}_{a}^{\mathcal{P}}$ of the inhomogeneous equation (42). By equations (42), (43) and (48) these discrete Wilson parameters correspond to the non-vanishing vectors $\widehat{\mathbf{v}}_{a}, \widetilde{\mathbf{v}}_{a}$ and $\widehat{\mathbf{v}}_{m}$. And these are related to the matrix elements $M_{i}{ }^{A}$ of the twist $\Theta$. Furthermore, equation (19) shows that our freedom in choosing $M_{i}{ }^{A}$ and hence discrete Wilson parameters, reflects our freedom in defining the gauge twist $\theta^{\prime}$. This shows that discrete Wilson parameters should not be regarded as moduli (which desribe continuous families of models with the same twist) but as parts of the definition of the gauge twist. This interpretation is familiar when the gauge twist is realized by shifts: In this case the Wilson lines are purely discrete, because $\mathrm{N}$ times a Wilson line must lie in the lattice $\Gamma_{16}$ and their effect on the massless spectrum spectrum is to impose extra projections, which eliminate all states that are no "Wilson singletts". Thus adding discrete Wilson lines is equivalent to modifying the twist by extra shift vectors [23].

\footnotetext{
10 Applying the same procedure to the $\widehat{\mathbf{A}}_{a}$ would lead to the trivial identity $0=0$, giving no discretizing constraint.

${ }^{11}$ In this case the "direct argument" applies, because $\theta^{\prime} \widehat{\mathbf{A}}_{m}=\widehat{\mathbf{A}}_{m}$ implies $\widehat{\mathbf{A}}_{m}=0$.
} 
The allowed set of values for the discrete Wilson lines in our construction is equal to that of the shift realization, if we restrict our attention to those twists, which admit the decomposition

$$
\left(\theta_{i}^{j}\right)=\left(\begin{array}{cc}
\theta_{a}^{b} & 0 \\
0 & \delta_{m}^{n}
\end{array}\right),
$$

w.r.t. a lattice basis. Geometrically, this is the case, when the twist acts in such a way, that fixtori (if they appear) factorize, such that the orbifold can be written as a product of a torus and an orbifold without fixed tori. (This is however not possible in general.) Using also that $\Gamma_{16}$ is selfdual, we can rewrite (19) as

$$
\mathbf{e}_{A} \cdot\left(\theta^{\prime} \mathbf{A}_{a}-\theta_{a}^{b} \mathbf{A}_{b}\right) \in \mathbf{Z}, \quad \mathbf{e}_{A} \cdot\left(\theta^{\prime} \mathbf{A}_{m}-\delta_{m}^{n} \mathbf{A}_{n}\right) \in \mathbf{Z}, \quad \forall A, a, m .
$$

Using the fact that

$$
\Pi_{1}=\frac{1}{N} \sum_{N=1}^{N} \theta^{\prime n}
$$

projects onto the invariant subspace and applying again the iterative trick, we get

$$
\mathbf{e}_{A} \cdot N \widetilde{\mathbf{A}}_{a} \in \mathbf{Z}, \quad \mathbf{e}_{A} \cdot N \widehat{\mathbf{A}}_{m} \in \mathbf{Z}, \quad \forall A, a, m .
$$

Since $\widetilde{\mathbf{A}}_{a}$ is invariant under $\theta^{\prime}$, and $\widehat{\mathbf{A}}_{m}$ orthogonal to the invariant subspace of $\theta^{\prime}$ this is equivalent to

$$
N \widetilde{\mathbf{A}}_{a} \in I_{\Gamma_{16}}:=\left\{\mathbf{w} \in \Gamma_{16} \mid \theta^{\prime} \mathbf{w}=\mathbf{w}\right\}, \quad \forall a,
$$

and

$$
N \widehat{\mathbf{A}}_{m} \in N_{\Gamma_{16}}:=\left\{\mathbf{w} \in \Gamma_{16} \mid \mathbf{w} \cdot I_{\Gamma_{16}}=0\right\}, \quad \forall m,
$$

where $I_{\Gamma_{16}}$ is the non-invariant sublattice of $\Gamma_{16}$ and $N_{\Gamma_{16}}$ its orthogonal complement. These constraints for discrete Wilson lines resemble those for the purely discrete Wilson lines in the case of shift realization of the gauge twist [6]. This adds further evidence to the claim that this construction yields only special points in the complete heterotic orbifold moduli space.

\subsection{From the D-matrix to the number of moduli (Part I)}

In this section we will start to derive the number of moduli, which is (almost) given by equation (20). After plugging (19) into (20), we get

$$
D_{i j} \theta_{k}^{j}-\theta_{i}^{j} D_{j k}=M_{i k}-\left(\theta^{\prime} \mathbf{A}_{i}-\theta_{i}^{j} \mathbf{A}_{j}\right) \cdot \mathbf{A}_{k} .
$$

Our goal is to solve this for the matrix $\mathbf{D}=\left(D_{i j}\right)$. The first step is to convert the left side of the equation into a proper commutator of matrices. This can be done by referring to an orthonormal basis instead of a lattice basis. This basis can of cause be chosen in a way that the decomposition (41) holds. The equation is now

$$
D_{\mu \rho} \theta_{\rho \nu}-\theta_{\mu \rho} D_{\rho \nu}=M_{\mu \nu}-\left(\theta^{\prime} \mathbf{A}_{\mu}-\theta_{\mu \rho} \mathbf{A}_{\rho}\right) \cdot \mathbf{A}_{\nu}
$$

with

$$
D_{\mu \nu}=T_{\mu}^{i} D_{i j} T_{\nu}^{j}, \quad M_{\mu \nu}=T_{\mu}^{i} M_{i j} T_{\nu}^{j},
$$

where, in terms of the matrix $\mathbf{T}=\left(T_{i}{ }^{\mu}\right)$ introduced in section 4.1:

$$
\left(T_{\mu}^{i}\right)=\mathbf{T}^{-1}, \quad\left(T_{\mu}^{i}\right)=\mathbf{T}^{T,-1} .
$$

\footnotetext{
${ }^{12}$ The definitions of $\mathbf{A}_{\mu}$ and $\theta_{\mu \nu}$ were given in section 4.1.
} 
The second step is to get rid of the second, Wilson line dependent term on the right hand side. Using (41) we observe that the discrete Wilson lines can be absorbed by a redefinition of the matrix $M_{\mu \nu}$, whereas the continuous Wilson lines can be absorped by the matrix $D_{\mu \nu}$ :

$$
\begin{gathered}
D_{a c} \theta_{c b}-\theta_{a c} D_{c b}=\underbrace{M_{a b}-\left(\widetilde{\mathbf{A}}_{a}-\theta_{a c} \widetilde{\mathbf{A}}_{c}\right) \cdot \widetilde{\mathbf{A}}_{b}}_{M_{a b}^{\prime}} . \\
D_{m n} \delta_{n p}-\delta_{m n} D_{n p}=0=\underbrace{M_{m p}-\left(\theta^{\prime} \widehat{\mathbf{A}}_{m}-\widehat{\mathbf{A}}_{m}\right) \cdot \widehat{\mathbf{A}}_{p}}_{M_{m p}^{\prime}} . \\
\underbrace{\left(D_{a m}+\widetilde{\mathbf{A}}_{a} \cdot \widetilde{\mathbf{A}}_{m}\right)}_{D_{a m}^{\prime}} \delta_{m n}-\theta_{a b} \underbrace{\left(D_{b n}+\widetilde{\mathbf{A}}_{b} \cdot \widetilde{\mathbf{A}}_{n}\right)}_{D_{b n}^{\prime}}=M_{a n} . \\
\underbrace{\left(D_{m c}+\left(\widehat{\mathbf{A}}_{m} \cdot \widehat{\mathbf{A}}_{c}\right)\right)}_{D_{m c}^{\prime}} \theta_{c b}-\delta_{m n} \underbrace{\left(D_{n b}+\left(\widehat{\mathbf{A}}_{n} \cdot \widehat{\mathbf{A}}_{b}\right)\right)}_{D_{n b}^{\prime}}=M_{m b} .
\end{gathered}
$$

Summarizing in matrix notation, with $\overline{\mathbf{D}}^{\prime}=\left(D_{\mu \nu}^{\prime}\right)$ and $\overline{\mathbf{M}}^{\prime}=\left(M_{\mu \nu}^{\prime}\right)$, we have transformed (20) into the inhomogeneous linear matrix equation

$$
\left[\overline{\mathbf{D}}^{\prime}, \bar{\vartheta}\right]=\overline{\mathbf{M}}^{\prime}
$$

To solve it, we need a particular solution of the inhomogeneous equation and the general solution of the corresponding homogeneous equation. This problem has been solved in reference [21] for purely symmetric and purely antisymmetric matrices ${ }^{13}$. The formula found for the particular solution also applies to the general case. The resulting matrix $\overline{\mathbf{D}}^{\mathcal{P}}$ consists of discrete parameters which reflect our freedom of chosing the matrix elements $M_{i k}$ of the twist $\Theta$. Like the discrete Wilson parameters discussed in the last section, they can be regarded as part of the definition of the twist. We will not analyze them further, but instead focus on the moduli.

The general solution of the homogeneous equation

$$
\left[\overline{\mathbf{D}}^{\prime}, \bar{\vartheta}\right]=0
$$

can be found by generalizing the calculation of 21] to the case of an arbitrary matrix: Remembering that the matrix $\bar{\vartheta}$ of the target space twist $\theta$ w.r.t. an orthonormal basis is an orthogonal matrix with $\bar{\vartheta}^{N}=\mathbf{1}$ we can transform it by an orthogonal transformation to its standard form

$$
\bar{\vartheta}=\left(\begin{array}{ccc}
\tau & \mathbf{0} & \mathbf{0} \\
\mathbf{0} & -\mathbf{1}_{l} & \mathbf{0} \\
\mathbf{0} & 0 & \mathbf{1}_{M}
\end{array}\right),
$$

where $\tau$ is an orthogonal $2 n \times 2 n$ matrix, $2 n+l+M=d$, and its eigenvalues are $\mathrm{N}$-th root of unity, but not \pm 1 . The matrix $\tau$ can be brought to the standard form

$$
\tau=\left(\begin{array}{ccc}
\tau_{1} & \cdots & \mathbf{0} \\
\vdots & \ddots & \vdots \\
\mathbf{0} & \cdots & \tau_{n}
\end{array}\right) .
$$

\footnotetext{
${ }^{13}$ In 21] consistency conditions for bosonic orbifolds were analyzed, with the result, that deformations of the compactification lattice, parametrized by a symmetric matrix $\mathbf{S}$, and the background field, described by an antisymmetric matrix $\mathbf{B}$ must commute with the target space twist. In case of the antisymmetric background field this must only hold up to a constant matrix.
} 
where the $2 \times 2$ blocks $\tau_{\alpha}$ correspond to complex conjugated pairs of eigenvalues $\exp \left( \pm 2 \pi i k_{\alpha}\right)$, $0<k_{\alpha}<1, k_{\alpha} \neq \frac{1}{2}, N k_{\alpha}=1, \alpha=1, \ldots, n$ :

$$
\tau_{\alpha}=\left(\begin{array}{cc}
c_{\alpha} & -s_{\alpha} \\
s_{\alpha} & c_{\alpha}
\end{array}\right), \quad c_{\alpha}=\cos \left(2 \pi k_{\alpha}\right), s_{\alpha}=\sin \left(2 \pi k_{\alpha}\right) .
$$

Plugging this special form of $\bar{\vartheta}$ into (66) yields

$$
\overline{\mathbf{D}}^{\prime}=\left(\begin{array}{ccc}
\mathrm{d} & \mathbf{0} & 0 \\
0 & \mathrm{p} & 0 \\
0 & 0 & \mathrm{q}
\end{array}\right) .
$$

The $l \times l$ matrix $\mathbf{p}$ and the $M \times M$ matrix $\mathbf{q}$ are arbitrary, whereas the $2 n \times 2 n$ matrix $\mathbf{d}$ must commute with $\tau$ :

$$
[\mathbf{d}, \tau]=\mathbf{0} .
$$

Decomposing $\mathbf{d}$ into $n^{2} 2 \times 2$ blocks $\mathbf{D}_{\alpha \beta}, \alpha, \beta=1, \ldots, n$, the equation reduces to

$$
\mathbf{D}_{\alpha \beta} \tau_{\beta}-\tau_{\alpha} \mathbf{D}_{\alpha \beta}=0 \quad \forall \alpha, \beta .
$$

If we set for arbitrary but fixed $\alpha, \beta$

$$
\mathbf{D}_{\alpha \beta}=\left(\begin{array}{cc}
p & q \\
r & t
\end{array}\right)
$$

we get a set of four homogeneous, linear equations in four real variables $p, q, r, t$. The necessary and sufficent condition for the existence of a nontrivial solution is the vanishing of the characteristic determinant of that system. Using identities for trigonometric functions this reduces to

$$
c_{\alpha}=c_{\beta},
$$

which implies

$$
s_{\alpha}= \pm s_{\beta} .
$$

Therefore equation (72) has two types of solutions:

- If $s_{\alpha}=s_{\beta}$, which is equivalent to $k_{\alpha}=k_{\beta}$ :

$$
q=-r, \quad p=t .
$$

- If $s_{\alpha}=-s_{\beta}$, which is equivalent to $k_{\beta}=1-k_{\alpha}$ :

$$
q=r, \quad p=-t .
$$

Thus each nonvanishing $\mathbf{D}_{\alpha \beta}$ yields two continuous parameters, whereas the unconstrained matrices $\mathbf{p}$, q give another $M^{2}+l^{2}$ parameters. Denoting the dimension of the eigenspace to eigenvalue $\exp (2 \pi i k)$ by $d_{k}$, the number of independent solutions of the homogeneous equation (66) is

$$
M_{D}=d_{1}^{2}+d_{\frac{1}{2}}^{2}+2 \sum_{k \in \mathbf{Q} \mid 0<k<\frac{1}{2}} d_{k}^{2}=\sum_{k \in \mathbf{Q} \mid 0 \leq k<1} d_{k}^{2} .
$$

This formula looks like a generalization of the formulas given in [21 for the metric and axionic moduli of bosonic orbifolds. However, it does not give the number of moduli directly. In order to identify the moduli in the heterotic case, we have to decompose the matrix $D_{\mu \nu}^{\prime}$ into the background fields. But if we want to give explicit formulas for the Wilson moduli, we must specify the gauge twist first. 


\subsection{Definition of the gauge twist}

According to equation (20) the Wilson lines must transform up to lattice vectors of $\Gamma_{16}$ under the gauge twist as the corresponding directions of target space transform under the target space twist. This resembles the embedding of the spin connection into the gauge connection used in Calabi-Yau compactifications and suggests that "somehow" the gauge twist is "identical" to the target space twist. This can be made precise in two steps 14: First we define the gauge twist by lifting the target space twist. To do this, we select a $d$-dimensional sublattice $\Lambda^{\prime}$ of $\Gamma_{16}$ with lattice basis $\widehat{\mathbf{e}}_{i}$, such that a bijective map (isomorphism of groups)

$$
\phi: \Lambda \rightarrow \Lambda^{\prime} \subset \Gamma_{16}: \mathbf{e}_{i} \rightarrow \widehat{\mathbf{e}}_{i}
$$

exists. Then we decompose the sixteen dimensional space spanned by $\Gamma_{16}$

$$
\left\langle\Gamma_{16}\right\rangle_{\mathbf{R}}=\left\langle\Lambda^{\prime}\right\rangle_{\mathbf{R}} \oplus\left\langle\Lambda^{\prime}\right\rangle_{\mathbf{R}}^{\perp}
$$

and define the gauge twist by

$$
\theta^{\prime}=\phi \theta \phi^{-1} \oplus \mathbf{1} .
$$

The second step is a variant of embedding the target space translation group into the gauge twist group [6. This is usually done by a map between the lattice basis of $\Lambda$ and the Wilson lines,

$$
\chi: \Lambda \rightarrow\left\langle\Gamma_{16}\right\rangle_{\mathbf{R}}: \mathbf{e}_{i} \rightarrow \mathbf{A}_{i}
$$

which must commute with the twist:

$$
\theta^{\prime} \chi=\chi \theta
$$

This implies

$$
\mathbf{A}_{i}=\theta_{i}^{j} \mathbf{A}_{j}
$$

which is equivalent to $\mathbf{v}_{i}=0$ or $M_{i}{ }^{A}=0$, implying that the Wilson lines must strictly transform like the corresponding directions in target space. This is, however, to restrictive in the context of our construction, because it does not allow for discrete Wilson lines $\left(\widetilde{\mathbf{v}}_{a}=\mathbf{0}\right.$ and $\left.\widehat{\mathbf{v}}_{m}=\mathbf{0}\right)$, which are, from our point of view, parts of the definition of the twist, and no (continuous) background fields. Therefore, we will only apply the milder constraint $\widehat{\mathbf{v}}_{a}=\mathbf{0}$ : This allows discrete Wilson lines $\widetilde{\mathbf{A}}_{a}, \widehat{\mathbf{A}}_{m}$, whereas the continuous Wilson lines transform strictly like the corresponding directions:

$$
\theta^{\prime} \widehat{\mathbf{A}}_{a}=\theta_{a b} \widehat{\mathbf{A}}_{b}, \quad \theta^{\prime} \widehat{\mathbf{A}}_{m}=\delta_{m n} \widehat{\mathbf{A}}_{n}=\widehat{\mathbf{A}}_{m} .
$$

Thus our modification amounts to applying the embedding (82) only to the continuous (parts of the) Wilson lines.

\subsection{From the D-matrix to the number of moduli (Part II)}

We are now ready to calculate the number of moduli. First let us decompose the matrix $\overline{\mathbf{D}}=\left(D_{\mu \nu}\right)$ into the background fields:

$$
D_{\mu \nu}=2\left(B_{\mu \nu}-G_{\mu \nu}-\frac{1}{4}\left(\mathbf{A}_{\mu} \cdot \mathbf{A}_{\nu}\right)\right) .
$$

Note that the metric moduli, which describe the allowed deformations of $\Lambda$ and can be parametrized by the lattice metric $G_{i j}$ do not appear in the D-matrix, when it is refered to an orthonormal basis, since

$$
G_{\mu \nu}=e_{\mu} \cdot e_{\nu}=\delta_{\mu \nu} .
$$

They can however be calculated from the constraint $\theta \in \operatorname{Aut}(\Lambda)$, as will be shown below. Now, the antisymmetric and symmetric part of $\overline{\mathbf{D}}$ correspond to the axionic moduli and to some

\footnotetext{
${ }^{14}$ We will see later, that this construction, together with a modified version, gives in the case of $\mathbf{Z}_{3}$ orbifolds based on $E_{8} \otimes E_{8}$ models all modular invariant theories. Thus it seems not to be very restrictive.
} 
Wilson moduli, parametrizing those deformations of the Wilson lines which change the matrix of scalar products $\left(\mathbf{A}_{\mu} \cdot \mathbf{A}_{\nu}\right)$ and will therefore be called metric Wilson moduli. There will also be isometric Wilson moduli describing orthogonal transformations, because rotations of the Wilson lines relative to the basis $\mathbf{e}_{A}$ of $\Gamma_{16}$ change the lattice $\Gamma_{16+d ; d}$ and therefore the physical spectrum.

To get the desired formulas, let us first assume that the target space twist has no fixtori $\left(d_{1}=0\right)$. Then the allowed Wilson lines are $\widehat{\mathbf{A}}_{a}, \widetilde{\mathbf{A}}_{a}, a=1, \ldots, d$, where the $\widehat{\mathbf{A}}_{a}$ take continuous values in the $d$-dimensional subspace $\left\langle\Lambda^{\prime}\right\rangle_{\mathbf{R}}$ and the $\widetilde{\mathbf{A}}_{a}$ take discrete values in $\left\langle\Lambda^{\prime}\right\rangle_{\mathbf{R}}{ }_{\mathbf{R}}$. In this case there is no difference between the original matrix $\left(D_{a b}\right)$ and the redefined matrix $\left(D_{a b}^{\prime}\right)$, and (78) gives the sum of the number $M_{B}$ of axionic moduli and the number $M_{A, m}$ of metric Wilson moduli. In order to separate them, we have to impose the extra constraint of antisymmetry or symmetry on the D-matrix. The result is (see also [21]) that both the antisymmetric and the symmetric part contain half of the moduli corresponding to the complex eigenvalues. As the part correponding to the eigenvalues -1 (the matrix $\mathbf{p}$ in $(70)$ ) is unconstrained, we get:

$$
\begin{aligned}
M_{B} & =\left(\begin{array}{c}
d_{\frac{1}{2}} \\
2
\end{array}\right)+\sum_{k \in \mathbf{Q} \mid 0<k<\frac{1}{2}} d_{k}^{2} . \\
M_{A, m} & =\left(\begin{array}{c}
d_{\frac{1}{2}}+1 \\
2
\end{array}\right)+\sum_{k \in \mathbf{Q} \mid 0<k<\frac{1}{2}} d_{k}^{2} .
\end{aligned}
$$

The number of axionic moduli is the same as in the bosonic case [21.

The number $M_{G}$ of metric moduli can now be extracted from the condition $\theta \in \operatorname{Aut}(\Lambda)$ in the same way as in bosonic orbifold models [21]. Let $\sigma$ be an invertible map deforming $\Lambda$ into another lattice $\Lambda^{\prime}$ :

$$
\sigma: \Lambda \rightarrow \Lambda^{\prime}: \mathbf{e}_{i} \rightarrow \mathbf{e}_{i}^{\prime}
$$

This mapping must not be confused with the coordinate transformation $\mathbf{e}_{i} \rightarrow \mathbf{e}_{\mu}$ introduced earlier. A deformation of $\Lambda$ is compatible with a given target space twist, if and only if $\theta$ is also an automorphism of $\Lambda^{\prime}$. Therefore the deformation must commute with the twist:

$$
\sigma \theta=\theta \sigma
$$

Since the lattice $\Lambda$ enters physics only through scalar products, only those deformations changing the lattice metric are moduli:

$$
G_{i j}=\mathbf{e}_{i} \cdot \mathbf{e}_{j} \rightarrow G_{i j}^{\prime}=\mathbf{e}_{i}^{\prime} \cdot \mathbf{e}_{j}^{\prime}
$$

This implies that we must consider symmetric maps only ${ }^{\text {[5 }}$. Referring to an orthonormal basis, such a map can be represented by a symmetric matrix $\mathbf{S}$. The resulting matrix equation

$$
[\overline{\mathbf{S}}, \bar{\vartheta}]=0
$$

is formally equivalent to that for the metric Wilson moduli, implying

$$
M_{G}=M_{A, m} .
$$

The number $M_{A, i}$ of isometric Wilson moduli can be calculated similarly 1 . Let $\rho$ be an orthogonal transformation acting non-trivially on the non-invariant subspace of $\left\langle\Gamma_{16}\right\rangle_{\mathbf{R}}$ and as the identity on the invariant subspace. Let $\mathbf{A}_{a}$ be Wilson lines which solve equations (38) and (58). Since the target space twist has by assumption no fixtori, all six Wilson lines can be

\footnotetext{
15 Remember that an invertible map can be decomposed into an orthogonal and a symmetric map by a polar decomposition.

${ }^{16}$ The argument given here in the first version of the paper misses the point under consideration. The author thanks Gabriel Lopes Cardoso for pointing out that the total number of Wilson moduli was to small in the case of degenerate eigenvalues of the twist.
} 
varied continuously on the non-invariant subspace $\left\langle\Lambda^{\prime}\right\rangle_{\mathbf{R}}$ and are discrete on the complement. If we deform the continuous parts $\widehat{\mathbf{A}}_{a}$ of the Wilson lines by $\rho$, then the new Wilson lines 17 $\rho \mathbf{A}_{a}$ are automatically solutions of (58), because $\rho$ is orthogonal. And they are solutions of (38) if and only if the deformation $\rho$ commutes with the gauge twist $\theta^{\prime}$ :

$$
\theta^{\prime} \rho=\rho \theta^{\prime} .
$$

Since $\rho$ is a continuous deformation of the old Wilson lines and therefore continuously connected to the identity, its matrix w.r.t. a orthonormal frame is in $S O(d)$ and can be written as the exponential of an antisymmetric matrix:

$$
\bar{R}=\exp (a), \quad a=-a^{t} \in \operatorname{so}(d) .
$$

Thus equation (95) is formally equivallent to the equation which gives the number of axionic moduli:

$$
\left[\bar{\vartheta}^{\prime}, \bar{R}\right]=0 \Leftrightarrow\left[\bar{\vartheta}^{\prime}, a\right]=0 .
$$

This implies that the number of isometric Wilson moduli equals the number of axionic moduli:

$$
M_{A, i}=M_{B} .
$$

The total number of Wilson moduli in the case without fixtori is:

$$
M_{A}=M_{A, m}+M_{A, i}=M_{G}+M_{B}=M_{D} .
$$

Let us now study what changes, when the target space twist has a $M$-dimensional invariant subspace $\left(M<d, d_{1}=M\right)$. Decomposing it as

$$
\theta=\widehat{\theta} \oplus \mathbf{1}_{M}
$$

we observe that the invariant subspace of the gauge twist also grows:

$$
\theta^{\prime}=\phi \widehat{\theta} \phi^{-1} \oplus \mathbf{1}_{M} \oplus \mathbf{1}_{16-d}=\phi \widehat{\theta} \phi^{-1} \oplus \mathbf{1}_{16-d+M}
$$

There are two kinds of continuous Wilson lines: $d-M$ Wilson lines $\widehat{\mathbf{A}}_{a}$ taking values in the $d-M$ dimensional non-invariant subspace $\widehat{V}$ of $\left\langle\Gamma_{16}\right\rangle_{\mathbf{R}}$ and $M$ Wilson lines $\widetilde{\mathbf{A}}_{m}$ taking values in the $16-d+M$ dimensional invariant subspace $\widetilde{V}$. As there are also discrete Wilson lines $\widetilde{\mathbf{A}}_{a}, \widehat{\mathbf{A}}_{m}$, we must take into account the additional off diagonal blocks which distinguish the modified matrix $D_{\mu \nu}^{\prime}$ from $D_{\mu \nu}$. By $(70)$ the equations for the off diagonal blocks are

$$
D_{a m}^{\prime}=-2 B_{a m}-\frac{1}{2} \widetilde{\mathbf{A}}_{a} \cdot \widetilde{\mathbf{A}}_{m}=0
$$

and

$$
D_{m a}^{\prime}=-2 B_{m a}-\frac{1}{2} \widehat{\mathbf{A}}_{m} \cdot \widehat{\mathbf{A}}_{a}=0 .
$$

Using the fact that scalar products are symmetric and that the B-matrix is antisymmetric, adding both equations gives:

$$
\widetilde{\mathbf{A}}_{a} \cdot \widetilde{\mathbf{A}}_{m}+\widehat{\mathbf{A}}_{a} \cdot \widehat{\mathbf{A}}_{m}=\mathbf{A}_{a} \cdot \mathbf{A}_{m}=0
$$

which means that Wilson lines assigned to invariant and those assigned to non-invariant directions must be pairwise orthogonal. Subtracting the equations for the off diagonal blocks yields

$$
8 B_{a m}-\widetilde{\mathbf{A}}_{a} \cdot \widetilde{\mathbf{A}}_{m}+\widehat{\mathbf{A}}_{a} \cdot \widehat{\mathbf{A}}_{m}=0
$$

\footnotetext{
${ }^{17}$ Note that rotations of the Wilson lines relative to $\Gamma_{16}$ change the scalar products $\mathbf{A}_{a} \cdot \mathbf{e}_{A}$ and are therefore physically relevant.
} 
Eliminating the invariant parts of the Wilson lines we get

$$
4 B_{a m}=-4 B_{m a}=-\widehat{\mathbf{A}}_{a} \cdot \widehat{\mathbf{A}}_{m}
$$

This shows that we can arbitrarily vary the continuous Wilson lines $\widehat{\mathbf{A}}_{a}$, as long as we tune the B-matrix according to (106) and restrict the continuous Wilson lines $\widetilde{\mathbf{A}}_{m}$ such that (104) holds. This prescription gives $F \leq M(d-M)$ constraints for the $\widetilde{\mathbf{A}}_{m}$, the precise number depending on how many linear independent (especially non-vanishing) discrete Wilson lines are chosen. The $\widetilde{\mathbf{A}}_{a}$ remain unconstrained. This parametrization of the constraints and moduli associated with the off-diagonal blocks of the $\mathrm{D}$-matrix will turn out to be convenient.

The analysis of the metric and isometric moduli associated with the $\widehat{\mathbf{A}}_{a}$ runs now parallel to the discussion of continuous Wilson lines in models without fixtori, because the diagonal block $D_{a b}$ is not modified (and because there is no extra constraint). The result is

$$
\widehat{M}_{A}=d_{\frac{1}{2}}^{2}+2 \sum_{k \in \mathbf{Q} \mid 0<k<\frac{1}{2}} d_{k}^{2}
$$

Looking at the block $D_{m n}$ we see that for the metric Wilson moduli of the $\widetilde{\mathbf{A}}_{m}$ no constraints arise. As the twist acts as the identity in this subspace, any deformation of the continuous Wilson lines commutes with it. Thus the only restriction is the one resulting from the off diagonal blocks of $D_{\mu \nu}^{\prime}$ :

$$
\widetilde{M}_{A}=M(16-d+M)-F \geq M(16-d+M)-M(d-M)=M(16-2 d+2 M) .
$$

The modifications of the formulas for the metric and axionic moduli are derived by observing that there are no constraints for the $\mathbf{q}$ block in (70). Taking into account that metric (axionic) deformations are symmetric (antisymmetric) we get:

$$
\begin{gathered}
M_{G}=\left(\begin{array}{c}
d_{1}+1 \\
2
\end{array}\right)+\left(\begin{array}{c}
d_{\frac{1}{2}}+1 \\
2
\end{array}\right)+\sum_{k \in \mathbf{Q} \mid 0<k<\frac{1}{2}} d_{k}^{2} . \\
M_{B}=\left(\begin{array}{c}
d_{1} \\
2
\end{array}\right)+\left(\begin{array}{c}
d_{\frac{1}{2}} \\
2
\end{array}\right)+\sum_{k \in \mathbf{Q} \mid 0<k<\frac{1}{2}} d_{k}^{2} .
\end{gathered}
$$

This completes the calculation of the number of moduli for all models with gauge twist defined by lifting of the target space twist.

\subsection{Multiple liftings}

A simple generalization of the definition of the gauge twist by lifting the target space twist is provided by liftings to different, orthogonal sublattices $\Lambda^{\prime}, \Lambda^{\prime \prime}, \ldots$ of $\Gamma_{16}$. If one compactifies six dimensions, there is, for dimensional reasons, only the possibility of simple and double liftings. In the second case there are two group isomorphisms

$$
\phi^{\prime}: \Lambda \rightarrow \Lambda^{\prime} \subset \Gamma_{16}, \quad \phi^{\prime \prime}: \Lambda \rightarrow \Lambda^{\prime \prime} \subset \Gamma_{16}, \quad \Lambda^{\prime} \perp \Lambda^{\prime \prime},
$$

and the gauge twist is

$$
\theta^{\prime}=\phi^{\prime} \theta \phi^{\prime-1} \oplus \phi^{\prime \prime} \theta \phi^{\prime \prime-1} \oplus \mathbf{1}_{4} .
$$

The generalization of the formulas derived for simple liftings should be straightforward. For example it is clear, that the number of moduli coming from continuous $\widehat{\mathbf{A}}_{a}$ Wilson lines is doubled. 


\section{The standard $\mathrm{Z}_{3}$ orbifold with continuous Wilson lines}

\subsection{Gauge twists for $\mathrm{Z}_{3}$ orbifolds}

The construction of $\mathbf{Z}_{3}$ orbifolds [5] is based on toroidal compactifications with the compactification lattice $\Lambda$ proportional to three copies of the root lattice of the Lie algebra $A_{2}$ (which is the complex form of $s u(3))$ :

$$
\Lambda \propto A_{2}^{\oplus 3}=A_{2} \oplus A_{2} \oplus A_{2}
$$

(We denote the root lattice with the same symbol as the complex Lie algebra.) The twist acts by simultanously rotating with an angle of 120 degree in all three $A_{2}$ lattices. This is the Coxeter twist ${ }^{18}$ of the corresponding Lie algebra. According to our formulas, there are 9 metric and 9 axionic moduli. The metric moduli correspond to three radii and six angles, since we can scale the three pairs of simple roots of the $A_{2}$ lattices and are also free to rotate the three planes spanned by them relative to each other, whereas the angle between the two simple roots of each $A_{2}$ is fixed.

The most basic constraint on the gauge twist is that it must be an autormophism of order three of the lattice $\Gamma_{16}$, which is chosen to be the $E_{8} \oplus E_{8}$ root lattice:

$$
\theta^{\prime} \in \operatorname{Aut}\left(E_{8} \oplus E_{8}\right), \quad \theta^{\prime 3}=1 .
$$

The Lie algebra $E_{8}$ has, up to conjugation, 112 automorphisms of finite order, which are listed in 25. They are all inner automorphisms and therefore induced by Weyl reflections of the root lattice. Among these, there are precisely four automorphisms of order three. These are induced by simultanous Coxeter twists in one, two, three or four pairwise orthogonal $A_{2}$ sublattices. Therefore, $E_{8} \oplus E_{8}$ has sixteen Weyl automorphisms of order three. However, modular invariance leads to constraints on the eigenvalues of the twist and only allows simultanous rotations in three or in six $A_{2}$ sublattices of the $E_{8} \oplus E_{8}$ lattice [7, 23]. Note that these cases singled out by modular invariance correspond precisely to simple or double liftings of the target space twist. Whether this observation is a pure coincidence or implies that the lifting construction of gauge twists is preferred is far from obvious. By different choices of the lift, there are altogether four inequivalent $\mathbf{Z}_{3}$ orbifold compactifications of the ten-dimensional $E_{8} \oplus E_{8}$ string theory [23]. The standard version is given by twisting a $A_{2}^{\oplus 3}$ sublattice of one $E_{8}$. This is the singular limit of a Calabi-Yau compactification with identification of the holonomy group $S U(3)$ with the $S U(3)$ subgroup of one $E(8)$ group [5, [1]. The unbroken gauge Lie algebra of the orbifold model without Wilson lines is then $E_{6} \oplus A_{2} \oplus E_{8}$. In the following we will study the effect of continuous and discrete Wilson lines on the Lie algebra. The first step is to introduce a convenient parametrization of the $E_{8}$ lattice, the gauge twist and the Wilson lines.

\subsection{Parametrization of the $E_{8}$ lattice}

Since the gauge twist is defined by its action on a $A_{2}^{\oplus 3}$ sublattice of one $E_{8}$ lattice, we will ignore the other $E_{8}$. We will use the following chain of maximal regular semisimple subalgebras 19 (and the corresponding root lattices):

$$
A_{2}^{\alpha} \oplus A_{2}^{\beta} \oplus A_{2}^{\gamma} \oplus A_{2}^{\epsilon} \subset E_{6}^{\delta} \oplus A_{2}^{\epsilon} \subset E_{8} .
$$

Each subalgebra is labled with some letter, which is also used to lable the simple roots, which spann the corresponding root lattice. Thus $\alpha_{i}, \beta_{i}, \gamma_{i}, \epsilon_{i}, i=1,2$ are simple roots of four (pairwise orthogonal) $A_{2}$ subalgebras, $\delta_{j}, j=1, \ldots, 6$ are simple roots of $E_{6}^{\delta}$ and $\mathbf{e}_{k}, k=1, \ldots, 8$ are simple roots of the $E_{8}$. We will also need the lowest roots $\delta_{0}, \mathbf{e}_{0}$ of $E_{6}^{\delta}$ and $E_{8}$. The corresponding fundamental weights are denoted by $\alpha_{i}^{*}, \ldots$. They are by definition dual to the roots:

$$
\alpha_{i}^{*} \cdot \alpha_{j}=\delta_{i j}, \ldots
$$

\footnotetext{
18 The Coxeter twist is the product of all fundamental Weyl reflections. For background material on Lie algebras see for example 24 .

${ }^{19}$ The relevant facts about subalgebras of simple Lie algebras can be found in 27]. For applications to symmetry breaking in toroidal compactifications, see [17].
} 


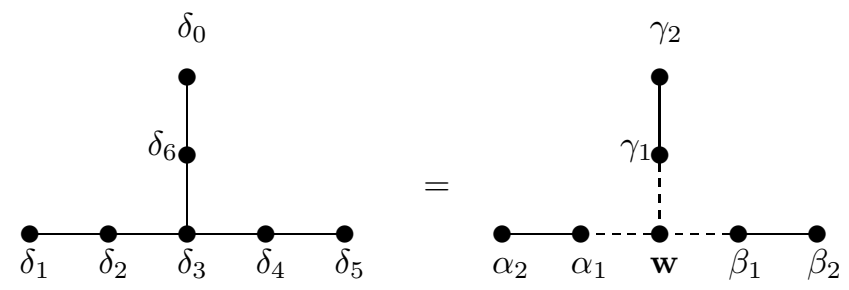

Figure 1: The extended Dynkin diagramm of $E_{6}$ is used to display the relations between the simple roots of $E_{6}$ and those of the subalgebra $A_{2}^{\oplus 3}$.

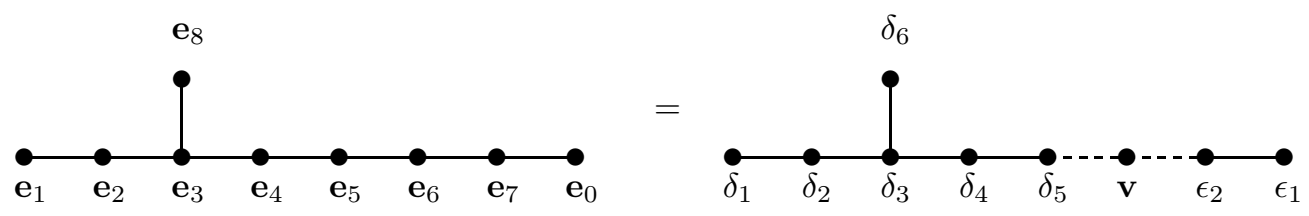

Figure 2: The extended Dynkin diagramm of $E_{8}$ is used to display the relations between the simple roots of $E_{8}$ and those of the subalgebra $E_{6} \oplus A_{2}$.

All algebraic relations between the roots ${ }^{20}$ are summarized by the extended root diagramms of $E_{6}^{\delta}$ and $E_{8}$. See figures 1 and 2 .

These diagramms make transparent, how the embeddings (115) work: The vectors

$$
\begin{gathered}
\mathbf{w}:=\delta_{3}=-\alpha_{1}^{*}-\beta_{1}^{*}-\gamma_{1}^{*} \\
\mathbf{v}:=\mathbf{e}_{6}=-\delta_{5}^{*}-\epsilon_{2}^{*}=-\beta_{2}^{*}+\gamma_{2}^{*}-\epsilon_{2}^{*}
\end{gathered}
$$

play a special role, because they "glue together" the semisimple Lie algebras $A_{2}^{\oplus 3}, E_{6} \oplus A_{2}$ to bigger simple ones $\left(E_{6}, E_{8}\right)$.

\subsection{Parametrization of the twist}

The gauge twist will be realized as a simultanuous Coxeter twist in $A_{2}^{\alpha}, A_{2}^{\beta}$ and $A_{2}^{\gamma}$ whereas $A_{2}^{\epsilon}$ (and of cause the second $E_{8}$ ) is invariant. The action of the twist on the simple roots of $A_{2}^{\alpha}$ is given by

$$
\alpha_{1} \longrightarrow \alpha_{2} \longrightarrow-\alpha_{1}-\alpha_{2} \longrightarrow \alpha_{1} \rightarrow \cdots
$$

The six simple roots $\pm \alpha_{1}, \pm \alpha_{2}$ und $\pm\left(\alpha_{1}+\alpha_{2}\right)$ form two orbits. Equivalent formulas hold for $A_{2}^{\beta}$ and $A_{2}^{\gamma}$. The fundamental weights $\alpha_{1}^{*}, \alpha_{2}^{*}$ are the highest weights of the $\mathbf{3}, \overline{\mathbf{3}}$ representations respectively. Their orbits under the twist give the other weights of these representations:

$$
\begin{aligned}
& \alpha_{1}^{*} \longrightarrow \alpha_{2}^{*}-\alpha_{1}^{*} \longrightarrow-\alpha_{2}^{*} \longrightarrow \alpha_{1}^{*} \rightarrow \cdots \\
& \alpha_{2}^{*} \longrightarrow \alpha_{1}^{*}-\alpha_{2}^{*} \longrightarrow-\alpha_{1}^{*} \longrightarrow \alpha_{2}^{*} \rightarrow \cdots
\end{aligned}
$$

As we have expressed the simple roots of the $E_{6}^{\delta}$ and $E_{8}$ in terms of the roots and weights of the $A_{2}^{\oplus 4}$ subalgebra, their behaviour under the twist is also clear.

\subsection{Parametrization of the Wilson lines}

According to our general results, there will be six continuous Wilson lines $\widehat{\mathbf{A}}_{i}, i=1, \ldots, 6$, taking values in the sixdimensional space spanned by the $A_{2}^{\alpha} \oplus A_{2}^{\beta} \oplus A_{2}^{\gamma}$ lattice:

$$
\widehat{V}=\left\langle A_{2}^{\alpha} \oplus A_{2}^{\beta} \oplus A_{2}^{\gamma}\right\rangle_{\mathbf{R}} \subset\left\langle E_{8} \oplus E_{8}\right\rangle_{\mathbf{R}}
$$

\footnotetext{
${ }^{20}$ To express the fundamental weights in terms of the roots, one has to use the inverse Cartan matrix.
} 
We will postpone the analysis of discrete Wilson lines, setting $\mathbf{A}_{i}=\widehat{\mathbf{A}}_{i}, \widetilde{\mathbf{A}}_{i}=0$. The Wilson lines must therefore strictly transform like the corresponding directions in target space and by the lifting prescription like the six simple roots of the $A_{2}^{\oplus 3}$ lattice:

$$
\mathbf{A}_{2 i-1} \longrightarrow \mathbf{A}_{2 i} \longrightarrow-\mathbf{A}_{2 i-1}-\mathbf{A}_{2 i} \longrightarrow \mathbf{A}_{2 i-1} \longrightarrow \cdots \quad i=1,2,3 .
$$

Our formulas precdict that there are nine metric and nine isometric Wilson moduli. These are easily identified. The geometric meaning of the metric Wilson moduli is the same as that of the metric moduli: One can scale three of six Wilson lines and can rotate the planes spanned by the three pairs of Wilson lines relative to each other. Thus one parametrization of the metric Wilson moduli is given by $\left|\mathbf{A}_{1}\right|,\left|\mathbf{A}_{3}\right|,\left|\mathbf{A}_{5}\right|,\left(\mathbf{A}_{1} \cdot \mathbf{A}_{3}\right),\left(\mathbf{A}_{1} \cdot \mathbf{A}_{4}\right),\left(\mathbf{A}_{1} \cdot \mathbf{A}_{5}\right),\left(\mathbf{A}_{1} \cdot \mathbf{A}_{6}\right),\left(\mathbf{A}_{3} \cdot \mathbf{A}_{5}\right)$, $\left(\mathbf{A}_{3} \cdot \mathbf{A}_{6}\right)$. The nine isometric Wilson moduli parametrize all rigid rotations of the six Wilson lines in $\widehat{V}$ that commute with the twist.

In this paper we will only study the nine metric Wilson moduli and keep the nine isometric Wilson moduli fixed. It will be convenient to parametrize the Wilson lines by weights of the $A_{2}^{\oplus 3}$ subalgebra in order to control the breaking of the gauge group. The coordinates on the nine dimensional subspace of the moduli space are the nine real numbers $p, q, r, x, y, z, a, b, c$, defined by:

$$
\begin{gathered}
\mathbf{A}_{1}=p \alpha_{1}^{*}+q \beta_{1}^{*}+r \gamma_{1}^{*}, \\
\mathbf{A}_{2}=p\left(\alpha_{2}^{*}-\alpha_{1}^{*}\right)+q\left(\beta_{2}^{*}-\beta_{1}^{*}\right)+r\left(\gamma_{2}^{*}-\gamma_{1}^{*}\right), \\
\mathbf{A}_{3}=x \alpha_{1}^{*}+y \beta_{1}^{*}+z \gamma_{1}^{*}, \\
\mathbf{A}_{4}=x\left(\alpha_{2}^{*}-\alpha_{1}^{*}\right)+y\left(\beta_{2}^{*}-\beta_{1}^{*}\right)+z\left(\gamma_{2}^{*}-\gamma_{1}^{*}\right), \\
\mathbf{A}_{5}=a \alpha_{1}^{*}+b \beta_{1}^{*}+c \gamma_{1}^{*}, \\
\mathbf{A}_{6}=a\left(\alpha_{2}^{*}-\alpha_{1}^{*}\right)+b\left(\beta_{2}^{*}-\beta_{1}^{*}\right)+c\left(\gamma_{2}^{*}-\gamma_{1}^{*}\right) .
\end{gathered}
$$

\subsection{A Strategy for the determination of the gauge group in orbifold models with continuous Wilson lines}

The gauge group of an orbifold model can be calculated in two steps. First one must know the gauge group of the underlying toroidal compactification as a function of the moduli, then one has to apply the twist in order to calculate the invariant subgroup. Whereas much is known about symmetry breaking and symmetry enhancement by Wilson lines in toroidal models [17, the explicit construction of the twist invariant subgroup is tedious and difficult. We will therefore try to determine it indirectly by combining our knowledge about the underlying torus compactification with some general facts about Lie algebras and their automorphisms. This will turn out to be sufficent in our example.

The toroidal model is completely specified by its momentum lattice. We will denote by $\Gamma$ the momentum lattice with no Wilson lines and generic (twist compatible) metric and axionic moduli. Its gauge Lie algebra will be $E_{8} \oplus E_{8} \oplus u(1)^{6}$, where the $u(1)^{6}$ comes from the six leftmoving internal oszillators $\alpha_{-1}^{i}$. Since our target space twist has no invariant subspace, this $u(1)^{6}$ will always be broken by the twist. Therefore it will be ignored in the following. The gauge twist is known ${ }^{21}$ to break the $E_{8} \oplus E_{8}$ to a $E_{6} \oplus A_{2} \oplus E_{8}$. If we switch on the nine twist compatible metric Wilson moduli, the original momentum lattice $\Gamma$ is deformed into another momentum lattice $\Gamma^{\prime}$. It is well known how to control what happens to the root vectors of the $E_{8} \oplus E_{8}$ : To each root $\mathbf{e}$ of $E_{8} \oplus E_{8}$, there corresponds a vector $\widehat{\mathbf{e}}$ in $\left\langle\Gamma^{\prime}\right\rangle_{\mathbf{R}}$,

$$
\widehat{\mathbf{e}}=\left(\mathbf{e}, \mathbf{0}_{d} ; \mathbf{0}_{d}\right),
$$

which is a lattice vector of $\Gamma^{\prime}$ if and only if it is an integer linear combinations of the lattice basis of $\Gamma^{\prime}$. As $\Gamma^{\prime}$ is selfdual, this is equivalent to $\widehat{\mathbf{e}}$ having integer scalar products with all basis vectors (5) - (7). The unbroken gauge Lie algebra of the deformed torus model is determined

\footnotetext{
${ }^{21}$ We will rederive this result in our formalim later.
} 


\begin{tabular}{|c|c|c|c|}
\hline type & Lie algebra, torus & Lie algebra, orbifold & moduli \\
\hline \hline$\alpha, \beta, \gamma$ & $A_{2} \oplus A_{2} \oplus A_{2}$ & $u(1)^{6}$ & 0 \\
\hline$\alpha, \beta$ & $A_{2} \oplus A_{2} \oplus u(1)^{2}$ & $u(1)^{4}$ & 3 \\
\hline$\alpha, \gamma$ & $A_{2} \oplus u(1)^{2} \oplus A_{2}$ & $u(1)^{4}$ & 3 \\
\hline$\beta, \gamma$ & $u(1)^{2} \oplus A_{2} \oplus A_{2}$ & $u(1)^{4}$ & 3 \\
\hline$\alpha$ & $A_{2} \oplus u(1)^{4}$ & $u(1)^{2}$ & 6 \\
\hline$\beta$ & $u(1)^{2} \oplus A_{2} \oplus u(1)^{2}$ & $u(1)^{2}$ & 6 \\
\hline$\gamma$ & $u(1)^{4} \oplus A_{2}$ & $u(1)^{2}$ & 6 \\
\hline- & $u(1)^{6}$ & - & 9 \\
\hline
\end{tabular}

Table 1: Summary of the effect of metric Wilson moduli on the $A_{2}^{\oplus 3}$ subalgebra. We display the type of the Wilson line, as defined in the text, the gauge symmetries of the toroidal and of the orbifold model, and the number of those moduli, which can still be varied.

by the subset of roots that remains in the lattice. As shown in 17 it is sufficent to control the simple roots. For generic values, all $E_{6}^{\delta}$ roots will not be in the deformed lattice. Thus the first $E_{8}$ is broken to $u(1)^{6} \oplus A_{2}^{\epsilon}$. Since the $u(1)^{6}$ comes from six internal oscillators, which transform non trivial under the gauge twist, this will be further broken to $A_{2}^{\epsilon}$ in the corresponding orbifold model. (The second $E_{8}$ can only be broken by discrete Wilson lines.)

To analyze the symmetry breakings induced by the nine metric Wilson moduli systematically, we will proceed in three steps. First we will study the effect on the sublattice $A_{2}^{\alpha} \oplus A_{2}^{\beta} \oplus A_{2}^{\gamma}$, then on $E_{6}^{\delta}$ and then on the complete first $E_{8}$.

\subsubsection{Step 1: Wilson lines on $A_{2}^{\oplus 3}$}

The vectors $\widehat{\alpha}_{i}$ corresponding to the simple roots $\alpha_{i}, i=1,2$ of $A_{2}^{\alpha}$ are elements of the lattice $\Gamma^{\prime}$ if and only if the parameters $p, x, a$ take simultanously integer values. This is seen by working out the scalar products between them and the lattice basis (5) - (7) using the parametrization (124) - (129) of the Wilson lines. If these three conditions are fulfilled, the gauge symmetry in the (corresponding sector of the) toroidal model is $A_{2}$ instead of $u(1)^{2}$. The six states corresponding to the six roots can be combined into eigenstates of the twist. Two of these combinations, namely the orbits of $\left|\alpha_{1}\right\rangle$ and $\left|-\alpha_{1}\right\rangle$ under the twist are invariant and therefore they are not projected out by the twist. Thus the twist breaks the $A_{2}$ to a $u(1)^{2}$. The dimension of the subspace where this happens is six, because we have to set three moduli to integer values. The corresponding critical Wilson lines will be called type- $\alpha$ Wilson lines.

Generalizing to the complete $A_{2}^{\oplus 3}$, there are also type- $\beta$ and type- $\gamma$ Wilson lines:

$$
\begin{aligned}
\text { type- } \alpha & : \Leftrightarrow \quad \widehat{\alpha}_{i} \in \Gamma^{\prime} \quad \Leftrightarrow \quad p, x, a \in \mathbf{Z} \\
\text { type- } \beta & : \Leftrightarrow \widehat{\beta}_{i} \in \Gamma^{\prime} \quad \Leftrightarrow \quad q, y, b \in \mathbf{Z} \\
\text { type- } \gamma & : \Leftrightarrow \widehat{\gamma}_{i} \in \Gamma^{\prime} \quad \Leftrightarrow \quad r, z, c \in \mathbf{Z}
\end{aligned}
$$

Combining the different conditions, we define type- $(\alpha, \beta)$ Wilson lines and so on. Summarizing all possible combinations we arrive at table 1. Geometrically the three critical three-dimensional subspaces intersect each other in discrete maximal critical points, and they are themselves the intersection loci of the three critical six-dimensional subspaces. 


\subsubsection{Step 2: Wilson lines on $E_{6}^{\delta}$}

In order to pass from the $A_{2}^{\oplus 3}$ subalgebra to the $E_{6}^{\delta}$, we have to study the effects of the Wilson lines and the twist on the simple root $\mathbf{w}$. Wilson lines, which are compatible with the corresponding vector $\widehat{\mathbf{w}}$ of the momentum lattice, will be called type-w Wilson lines:

$$
\text { type- } \mathbf{w}: \Leftrightarrow \widehat{\mathbf{w}} \in \Gamma^{\prime} \Leftrightarrow p+q+r \in 3 \mathbf{Z} \text { and } x+y+z \in 3 \mathbf{Z} \text { and } a+b+c \in 3 \mathbf{Z} .
$$

If the Wilson lines are not type- $\mathbf{w}$, then the symmetry of the toroidal model is at most $A_{2}^{\oplus 3}$ and the analysis of the last subsection applies. Let us then look at Wilson lines, which are type- $\mathbf{w}$, but not type $-\alpha,-\beta$ or $-\gamma$. In this case the vectors $\pm \widehat{\mathbf{w}}$ are in $\Gamma^{\prime}$. But the Wilson lines are compatible with the gauge twist, and all vectors belonging to the orbits of the two vectors must also be in $\Gamma^{\prime}$. These are, expressed as weights of the $A_{2}^{\oplus 3}$ subalgebra:

$$
\pm\left(-\widehat{\alpha}_{1}^{*}-\widehat{\beta}_{1}^{*}-\widehat{\gamma}_{1}^{*}\right), \quad \pm\left(\widehat{\alpha}_{1}^{*}-\widehat{\alpha}_{2}^{*}+\widehat{\beta}_{1}^{*}-\widehat{\beta}_{2}^{*}+\widehat{\gamma}_{1}^{*}-\widehat{\gamma}_{2}^{*}\right), \quad \pm\left(\widehat{\alpha}_{2}^{*}+\widehat{\beta}_{2}^{*}+\widehat{\gamma}_{2}^{*}\right) .
$$

Since they form the root system of a $A_{2}$, the symmetry of the torus model is enlarged from $u(1)^{6}$ to $A_{2} \oplus u(1)^{4}$. In the corresponding orbifold model the residual symmetry will be $u(1)^{2}$, because there are two twist invariant orbits from the six roots.

If the Wilson lines are type- $(\mathbf{w}, \alpha)$ the symmetry is enlarged further. Beside the $2 \cdot 6$ roots from the twist orbits of $\pm \alpha_{1}$ and $\pm \mathbf{w}$, we can construct another 12 roots. This is most easily seen by observing that all vectors in the orbits of $\pm \mathbf{w}$ contain a weight of the $\overline{\mathbf{3}}$ or $\mathbf{3}$ representations of $A_{2}^{\alpha}$. By adding a suitable root of $A_{2}^{\alpha}$ this weight can be replaced by any other weight of the

same representation without changing the $A_{2}^{\beta} \oplus A_{2}^{\gamma}$ part and the norm of the vector. Altogether there are 24 roots. The resulting Lie algebra must have rang 6 and be a regular subalgebra of $E_{6}^{\delta}$. Using Dynkins algorithm for regular subalgebras, one can show that there is only one such subalgebra, $D_{4} \oplus u(1)^{2}$. This is the enlarged symmetry of the torus model. The orbifold twist will break the $u(1)^{2}$ completely. The $D_{4}$ roots can be arranged into 8 invariant orbits. This counting shows that the Lie algebra of the twisted model is $A_{2}$ or $A_{1} \oplus A_{1} \oplus u(1)^{2}$. If the twist is an inner automorphism of $D_{4}$, it cannot reduce the rank and the $A_{2}$ is excluded. However there is no reason for the twist to be an inner automorphism: A twist of the root lattice of a Lie algebra does only define an inner automorphism if it is a Weyl twist. The outer automorphisms (up to inner ones) are in one to one correspondence with symmetries of the Dynkin diagram [27. The Lie algebra $D_{4}$ is the only simple Lie algebra with an outer automorphim of order three. As all other simple Lie algebra have outer automorphisms of at most order two, and we are studying a twist of order three, we do not know whether the automorphism is inner or outer if and only if the symmetry of the toroidal model is $D_{4}$.

Fortunately we can determine the rank of the twist invariant subalgebra by writing down all eight invariant combinations of Kac-Moody currents and calculating their operator product expansions. Then we see that the currents corresponding to the orbits of $\pm \alpha_{1}$ fulfill a $u(1)^{2}$ current algebra, and that their OPEs with the other six allways contain a first order pole. This shows that the rank of the orbifold Lie algebra is two. Thus the algebra is $A_{2}$ and the automorphism must be an outer one.

The cases of type $-(\mathbf{w}, \beta)$ and type- $-(\mathbf{w}, \gamma)$ Wilson lines are completely analog. The next step is to note that there are no type $-(\mathbf{w}, \alpha, \beta)$, type $-(\mathbf{w}, \alpha, \gamma)$ or type $-(\mathbf{w}, \beta, \gamma)$ Wilson lines, because the corresponding conditions cannot be fulfilled simultanously. There are however type- $(\mathbf{w}, \alpha, \beta, \gamma)$ Wilson lines. In this case all simple roots of $E_{6}^{\delta}$ are present. (Looking into the details, we find, that by adding $A_{2}^{\oplus 3}$ roots to the vectors in the orbits of $\pm \mathbf{w}$, all $2 \cdot 27$ weights of the representations $(\overline{\mathbf{3}}, \overline{\mathbf{3}}, \overline{\mathbf{3}})(\mathbf{3}, \mathbf{3}, \mathbf{3})$ can be constructed, thus extending the $A_{2}^{\oplus 3}$ root lattice to the $E_{6}$ root lattice.) To calculate the orbifold Lie algebra, it is sufficent to count the invariant orbits: Since there are 24 invariant states, and the automorphism must be inner, we must look for a regular rank six subalgebra of $E_{6}$ with dimension 24. Using Dynkins formalism we see that the unique solution is $A_{2}^{\oplus 3}$.

Summarizing, the possible breakings of $E_{6}$ by twist and continuous Wilson lines are collected in table 2. The number of residual moduli is again calculated by counting the conditions, that the Wilson moduli must fulfill. 


\begin{tabular}{|c|c|c|c|}
\hline Type & Lie algebra, torus & Lie algebra, orbifold & Moduli \\
\hline \hline $\mathbf{w}, \alpha, \beta, \gamma$ & $E_{6}$ & $A_{2} \oplus A_{2} \oplus A_{2}$ & 0 \\
\hline $\mathbf{w}, \alpha$ & $D_{4} \oplus u(1)^{2}$ & $A_{2}$ & 3 \\
\hline $\mathbf{w}, \beta$ & $D_{4} \oplus u(1)^{2}$ & $A_{2}$ & 3 \\
\hline $\mathbf{w}, \gamma$ & $D_{4} \oplus u(1)^{2}$ & $A_{2}$ & 3 \\
\hline $\mathbf{w}$ & $A_{2} \oplus u(1)^{4}$ & $u(1)^{2}$ & 6 \\
\hline
\end{tabular}

Table 2: Summary of the effect of type-w Wilson lines on the $E_{6}$ subalgebra. For Wilson lines, which are not type- $\mathbf{w}$, see table 1 .

\subsubsection{Step 3: Wilson lines on $E_{8}$}

The last step is to extend our analysis to the full $E_{8}$ lattice. As the sublattice $A_{2}^{\epsilon}$ is both twist invariant and orthogonal to the Wilson lines, the corresponding $A_{2}$ algebra will be unbroken in the toroidal and in the orbifold model. The only thing to do is to look at the lattice vector $\widehat{\mathbf{v}}$ corresponding to the simple root $\mathbf{v}$ of $E_{8}$ which extends $E_{6}^{\delta} \oplus A_{2}^{\epsilon}$ to $E_{8}$. This leeds to a new type of critical Wilson lines:

$$
\text { type- } \mathbf{v}: \Leftrightarrow \widehat{\mathbf{v}} \in \Gamma^{\prime} \Leftrightarrow(q-r) \in 3 \mathbf{Z} \text { and }(y-z) \in 3 \mathbf{Z} \text { and }(b-c) \in 3 \mathbf{Z} .
$$

Using the same methods as in the last subsection, one arrives at table 3 . Instead of going through all the cases, let us comment on the case of type- $\mathbf{v}$ Wilson lines. Here it is easily shown, that the unbroken Lie algebra of the torus model is $u(1)^{4} \oplus D_{4}$. By counting the invariant states we know that the Lie algebra of the corresponding orbifold has dimension 14. We also know that it has $A_{2}^{\epsilon}$ as a subalgebra and that the rank is between two and four. Using Dynkins algorithm it is easy to show, that there is no regular subalgebra of $D_{4}$ with these properties. It is however well know that $D_{4}$ has a non-regular (and also non simply laced) subalgebra $G_{2}$, which is the invariant subalgebra under a third order outer automorphism 22 . Therefore, this must be the unbroken Lie algebra in this case. All other cases in which similar complications arrise can be reduced to this case. For a summary see table 3 .

\subsection{The Effect of discrete Wilson lines}

Our last point is to study the effect of discrete Wilson lines on the gauge group. Again we will restrict our attention to the first $E_{8}$. According to the general results there are two constraints for discrete Wilson lines $\widetilde{\mathbf{A}}_{i}$ : The first is, that multiplying $\widetilde{\mathbf{A}}_{i}$ with the order of the twist must give an invariant lattice vector of the $E_{8}$ lattice. The sublattice of (pointwise) invariant lattice vectors is precisely the root lattice $A_{2}^{\epsilon}$ :

$$
3 \widetilde{\mathbf{A}}_{i} \in I_{E_{8}}=A_{2}^{\epsilon}
$$

The other constraint is, that the $\widetilde{\mathbf{A}}_{i}$ must transform, up to a lattice vector of $I_{E_{8}}$, as the corresponding directions transform under the target space twist:

$$
\theta^{\prime} \widetilde{\mathbf{A}}_{i}-\theta_{i}^{j} \widetilde{\mathbf{A}}_{j}=\left(\delta_{i}^{j}-\theta_{i}^{j}\right) \widetilde{\mathbf{A}}_{j} \in A_{2}^{\epsilon} .
$$

Using the explicit form of the twist we get

$$
\left\{\begin{array}{l}
\widetilde{\mathbf{A}}_{2 k-1}-\widetilde{\mathbf{A}}_{2 k} \in A_{2}^{\epsilon}, \\
\widetilde{\mathbf{A}}_{2 k-1}+2 \widetilde{\mathbf{A}}_{2 k} \in A_{2}^{\epsilon},
\end{array}\right.
$$

\footnotetext{
${ }^{22}$ This is used to construct the twisted affine Lie algebra $D_{4}^{(3)}$ 26].
} 
with $k=1,2,3$. Rearranging this to

$$
\begin{cases}3 \widetilde{\mathbf{A}}_{i} \in A_{2}^{\epsilon}, & i=1, \ldots, 6, \\ \widetilde{\mathbf{A}}_{2 k-1}-\widetilde{\mathbf{A}}_{2 k} \in A_{2}^{\epsilon}, & k=1,2,3,\end{cases}
$$

we learn that those discrete Wilson lines, which are connected by the target space twist, must be in the same coset of $\frac{1}{3} A_{2}^{\epsilon}$ with respect to $A_{2}^{\epsilon}$. There are nine such cosets. Their canonical representatives are

$$
\frac{m}{3} \epsilon_{1}+\frac{n}{3} \epsilon_{2}, \quad m, n=0,1,2 .
$$

To determine the effect on the gauge group, we note that three of these are weights of the Lie algebra $A_{2}^{\epsilon}$, namely

$$
\frac{2}{3} \alpha_{1}+\frac{1}{3} \alpha_{2}=\alpha_{1}^{*}, \quad \frac{1}{3} \alpha_{1}+\frac{2}{3} \alpha_{2}=\alpha_{2}^{*} \text { und } \mathbf{0} .
$$

whereas the other six are not. Concerning the gauge group there are only two possibilities: The first is that all six Wilson lines $\widetilde{\mathbf{A}}_{i}$ are weights of $A_{2}^{\epsilon}$. Then the $A_{2}^{\epsilon}$ roots are still present in the deformed lattice $\Gamma^{\prime}$. Thus the $A_{2}^{\epsilon}$ will not be broken in the torus model, and since the twist operates trivially on $A_{2}^{\epsilon}$, it is also unbroken in the twisted model. The gauge groups are the same as in the case without discrete Wilson lines and table 3 remains valid. These Wilson lines will be called type $-\epsilon$.

But if at least one pair of Wilson lines takes values in a different coset, then the $A_{2}^{\epsilon}$ is broken to a $u(1)^{2}$ in the torus model. Again, this is not further broken by the twist. In order to modify table 3 we have to look at the vector $\mathbf{v}$, because it is a non-trivial weight of $A_{2}^{\epsilon}$. To derive a condition for $\widehat{\mathbf{v}}$ to be in $\Gamma^{\prime}$, let us parametrize the discrete Wilson lines by their canonical coset representatives:

$$
\widetilde{\mathbf{A}}_{1} \simeq \widetilde{\mathbf{A}}_{2} \simeq \frac{m_{1}}{3} \epsilon_{1}+\frac{n_{1}}{3} \epsilon_{2}, \quad \widetilde{\mathbf{A}}_{3} \simeq \widetilde{\mathbf{A}}_{4} \simeq \frac{m_{2}}{3} \epsilon_{1}+\frac{n_{2}}{3} \epsilon_{2}, \quad \widetilde{\mathbf{A}}_{5} \simeq \widetilde{\mathbf{A}}_{6} \simeq \frac{m_{3}}{3} \epsilon_{1}+\frac{n_{3}}{3} \epsilon_{2} .
$$

The modified definition of a type- $\mathbf{v}$ Wilson line for nontrivial discrete Wilson lines is:

$$
\begin{gathered}
\text { type- } \mathbf{v}: \Leftrightarrow \widehat{\mathbf{v}} \in \Gamma^{\prime} \\
\Leftrightarrow\left(q-r-n_{1}\right) \in 3 \mathbf{Z} \text { and }\left(y-z-n_{2}\right) \in 3 \mathbf{Z} \text { and }\left(b-c-n_{3}\right) \in 3 \mathbf{Z} .
\end{gathered}
$$

We can now study the effect of the nine metric Wilson moduli with the same methods as before. The results are shown in table 1 .

\section{Conclusions}

Summarizing, we have identified the untwisted moduli of heterotic $\mathbf{Z}_{N}$ orbifold compactifications and developed a method to determine the gauge group, which was sucessfully applied to the standard $\mathbf{Z}_{3}$ orbifold. All constraints on the background fields were derived systematically from the basic constraint $\Theta \in \operatorname{Aut}\left(\Gamma_{16+d ; d}\right)$. By separating the continuous and discrete parts of the background fields, we have shown that the appearence of discrete background fields is due to the fact that the twist of the momentum lattice is not uniquely fixed by the target space twist. The choice of the gauge twist turned out to be highly restricted and the lifting and embedding construction used here seems to be the only natural solution. The geometry of critical subspaces found in the $\mathbf{Z}_{3}$ example is similar to that found in the case of toroidal compactifications in [17]. It should be emphasized, that the characterization of critical subspaces is independent of the determination of the gauge group. Thus even if it is an accident that we could get the gauge groups of the orbifold models without explicit construction, the geometry of critical subspaces should alway be accessible. And the result that the critical geometries of the two orbifold moduli spaces connected by choosing different discrete Wilson lines are in one to one correspondence with one another and with the underlying toroidal moduli spaces must also 


\begin{tabular}{|c|c|c|c|c|}
\hline Type & Lie algebra, torus & Lie algebra, orbifold & gauge group & Moduli \\
\hline \hline $\mathbf{v}, \mathbf{w}, \alpha, \beta, \gamma$ & $E_{8}$ & $E_{6} \oplus A_{2}$ & $E(6) \otimes S U(3)$ & 0 \\
\hline $\mathbf{v}, \alpha, \beta, \gamma$ & $A_{2} \oplus E_{6}$ & $D_{4} \oplus u(1)^{4}$ & $S O(8) \otimes U(1)^{2}$ & 0 \\
\hline $\mathbf{w}, \alpha, \beta, \gamma$ & $E_{6} \oplus A_{2}$ & $A_{2} \oplus A_{2} \oplus A_{2} \oplus A_{2}$ & $(S U(3))^{4}$ & 0 \\
\hline$\alpha, \beta, \gamma$ & $A_{2} \oplus A_{2} \oplus A_{2} \oplus A_{2}$ & $u(1)^{6} \oplus A_{2}$ & $S U(3) \otimes U(1)^{6}$ & 0 \\
\hline $\mathbf{v}, \mathbf{w}, \alpha$ & $D_{4} \oplus D_{4} \oplus u(1)^{2}$ & $A_{2} \oplus G_{2}$ & $G(2) \otimes S U(3)$ & 0 \\
\hline $\mathbf{v}, \mathbf{w}$ & $A_{2} \oplus D_{4} \oplus u(1)^{2}$ & $G_{2} \oplus u(1)^{2}$ & $G(2) \otimes U(1)^{2}$ & 3 \\
\hline $\mathbf{v}, \alpha$ & $A_{2} \oplus D_{4} \oplus u(1)^{2}$ & $G_{2} \oplus u(1)^{2}$ & $G(2) \otimes U(1)^{2}$ & 3 \\
\hline $\mathbf{v}, \beta, \gamma$ & $u(1)^{2} \oplus E_{6}$ & $D_{4} \oplus u(1)^{2}$ & $S O(8) \otimes U(1)^{2}$ & 3 \\
\hline$\alpha, \beta$ & $A_{2} \oplus A_{2} \oplus u(1)^{2} \oplus A_{2}$ & $u(1)^{4} \oplus A_{2}$ & $S U(3) \otimes U(1)^{4}$ & 3 \\
\hline$\alpha, \gamma$ & $A_{2} \oplus A_{2} \oplus u(1)^{2} \oplus A_{2}$ & $u(1)^{4} \oplus A_{2}$ & $S U(3) \otimes U(1)^{4}$ & 3 \\
\hline$\beta, \gamma$ & $A_{2} \oplus A_{2} \oplus u(1)^{2} \oplus A_{2}$ & $u(1)^{4} \oplus A_{2}$ & $S U(3) \otimes U(1)^{4}$ & 3 \\
\hline $\mathbf{w}, \alpha$ & $D_{4} \oplus u(1)^{2} \oplus A_{2}$ & $A_{2} \oplus A_{2}$ & $S U(3) \otimes S U(3)$ & 3 \\
\hline $\mathbf{w}, \beta$ & $D_{4} \oplus u(1)^{2} \oplus A_{2}$ & $A_{2} \oplus A_{2}$ & $S U(3) \otimes S U(3)$ & 3 \\
\hline $\mathbf{w}, \gamma$ & $D_{4} \oplus u(1)^{2} \oplus A_{2}$ & $A_{2} \oplus A_{2}$ & $S U(3) \otimes S U(3)$ & 3 \\
\hline $\mathbf{w}$ & $A_{2} \oplus u(1)^{4} \oplus A_{2}$ & $u(1)^{2} \oplus A_{2}$ & $S U(3) \otimes U(1)^{2}$ & 6 \\
\hline$\alpha$ & $A_{2} \oplus u(1)^{4} \oplus A_{2}$ & $u(1)^{2} \oplus A_{2}$ & $S U(3) \otimes U(1)^{2}$ & 6 \\
\hline$\beta$ & $A_{2} \oplus u(1)^{4} \oplus A_{2}$ & $u(1)^{2} \oplus A_{2}$ & $S U(3) \otimes U(1)^{2}$ & 6 \\
\hline$\gamma$ & $A_{2} \oplus u(1)^{4} \oplus A_{2}$ & $u(1)^{2} \oplus A_{2}$ & $S U(3) \otimes U(1)^{2}$ & 6 \\
\hline $\mathbf{v}$ & $D_{4} \oplus u(1)^{4}$ & $G_{2}$ & $G(2)$ & 6 \\
\hline- & $u(1)^{6} \oplus A_{2}$ & $A_{2}$ & $S U(3)$ & 9 \\
\hline
\end{tabular}

Table 3: In this table we summarize all possibilities of breaking the standard $\mathbf{Z}_{3}$ orbifold by nine metric Wilson moduli, but without discrete Wilson lines. We display the type of critical Wilson lines as explained in the text, the Lie algebras realized in the untwisted (toroidal) and twisted (orbifold) model, the unbroken gauge group of the orbifold model, and the number of moduli, that can still be varied continuously. 


\begin{tabular}{|c|c|c|c|c|}
\hline Type & Lie Algebra, torus & Lie Algebra, orbifold & gauge group & Moduli \\
\hline \hline $\mathbf{v}, \mathbf{w}, \alpha, \beta, \gamma$ & $E_{7} \oplus u(1)$ & $A_{5} \oplus A_{2} \oplus u(1)$ & $S U(6) \otimes S U(3) \otimes U(1)$ & 0 \\
\hline $\mathbf{v}, \alpha, \beta, \gamma$ & $A_{2} \oplus A_{5} \oplus u(1)$ & $u(1)^{6} \oplus A_{2}$ & $S U(3) \otimes U(1)^{6}$ & 0 \\
\hline $\mathbf{w}, \alpha, \beta, \gamma$ & $E_{6} \oplus u(1)^{2}$ & $A_{2} \oplus A_{2} \oplus A_{2} \oplus u(1)^{2}$ & $(S U(3))^{3} \otimes U(1)^{2}$ & 0 \\
\hline$\alpha, \beta, \gamma$ & $A_{2} \oplus A_{2} \oplus A_{2} \oplus u(1)^{2}$ & $u(1)^{8}$ & $U(1)^{8}$ & 0 \\
\hline $\mathbf{v}, \mathbf{w}, \alpha$ & $D_{4} \oplus A_{2} \oplus u(1)^{4}$ & $A_{2} \oplus u(1)^{2}$ & $S U(3) \otimes U(1)^{2}$ & 0 \\
\hline $\mathbf{v}, \mathbf{w}$ & $A_{2} \oplus A_{2} \oplus u(1)^{4}$ & $u(1)^{4}$ & $U(1)^{4}$ & 3 \\
\hline $\mathbf{v}, \alpha$ & $A_{2} \oplus A_{2} \oplus u(1)^{4}$ & $u(1)^{4}$ & $U(1)^{4}$ & 3 \\
\hline $\mathbf{v}, \beta, \gamma$ & $u(1)^{3} \oplus A_{5}$ & $A_{2} \oplus u(1)^{2}$ & $S U(3) \otimes U(1)^{2}$ & 3 \\
\hline$\alpha, \beta$ & $A_{2} \oplus A_{2} \oplus u(1)^{4}$ & $u(1)^{6}$ & $U(1)^{6}$ & 3 \\
\hline$\alpha, \gamma$ & $A_{2} \oplus A_{2} \oplus u(1)^{4}$ & $u(1)^{6}$ & $U(1)^{6}$ & 3 \\
\hline$\beta, \gamma$ & $A_{2} \oplus A_{2} \oplus u(1)^{4}$ & $u(1)^{6}$ & $U(1)^{6}$ & 3 \\
\hline $\mathbf{w}, \alpha$ & $D_{4} \oplus u(1)^{4}$ & $A_{2} \oplus u(1)^{2}$ & $S U(3) \otimes U(1)^{2}$ & 3 \\
\hline $\mathbf{w}, \beta$ & $D_{4} \oplus u(1)^{4}$ & $A_{2} \oplus u(1)^{2}$ & $S U(3) \otimes U(1)^{2}$ & 3 \\
\hline $\mathbf{w}, \gamma$ & $D_{4} \oplus u(1)^{2} \oplus u(1)^{2}$ & $A_{2} \oplus u(1)^{2}$ & $S U(3) \otimes U(1)^{2}$ & 3 \\
\hline $\mathbf{w}$ & $A_{2} \oplus u(1)^{6}$ & $u(1)^{4}$ & $U(1)^{4}$ & 6 \\
\hline$\alpha$ & $A_{2} \oplus u(1)^{6}$ & $u(1)^{4}$ & $U(1)^{4}$ & 6 \\
\hline$\beta$ & $A_{2} \oplus u(1)^{6}$ & $u(1)^{4}$ & $U(1)^{4}$ & 6 \\
\hline$\gamma$ & $A_{2} \oplus u(1)^{6}$ & $u(1)^{4}$ & $U(1)^{4}$ & 6 \\
\hline $\mathbf{v}$ & $A_{2} \oplus u(1)^{6}$ & $u(1)^{4}$ & & 9 \\
\hline- & $u(1)^{8}$ & $u(1)^{2}$ & & \\
\hline
\end{tabular}

Table 4: This table contains all possibilities of symmetry breaking of the standard $\mathbf{Z}_{3}$ orbifold in the case when the subalgebra $A_{2}^{\epsilon}$ is broken by discrete Wilson lines. The second $E_{8}$ is ignored. 
hold more generally. The dimensions and critical subspaces of different orbifold moduli spaces with the same target space twist but inequivalent discrete background fields should always be isomorphic.

There are also some implications concerning the modular groups for orbifolds with Wilson moduli. Since points of maximaly extended symmetry are usually fixpoints, we expect that critical subspaces of non maximal symmetry also transform somewhat singular, for example they could be invariant (not pointwise) or transform into one another. The construction of modular groups will be one of the next steps.

Note also that there are two further types of moduli, which should be included in the future. For orbifolds without Wilson lines it is known that there are moduli arrising from the twisted sectors (twisted moduli) which parametrize the blowing up of the orbifold singularities. If these can coexist with the Wilson moduli, this could be used to explore more general $(0,2)$ compactifications. It is also possible that at points of extended symmetry in the untwisted moduli space additional moduli arrise which correspond to extra (non-redundant) marginal operators. Since this is interelated with extended gauge symmetry and special behaviour under modular transformations, thess moduli should have a nice group theoretical characterisation, if they exist.

There is of course a lot of other things that can be studied now, for example the complete massless spectrum, the twisted sectors (where the degeneracy of the ground states is lifted by the Wilson lines [23]), the construction of vertex operators, the calculation of correlators, and the derivation of effective actions.

\section{References}

[1] M. Green, J. Schwarz, E. Witten, Superstring Theory. Cambridge University Press (1987), 2 volumes.

[2] M. Kaku, Strings, Conformal Fields and Topology. Springer (1991).

[3] R. Dijkgraaf, E. Verlinde, H. Verlinde, On Moduli Spaces of Conformal Field Theories with $c \geq 1$, in P. Di Vecchia (ed.) Perspektives in String Theories. World Scientific (1988).

[4] S. Ferrara, S. Theisen, Moduli Spaces, Effective Actions and Duality Symmetry in String Compactifications. CERN-Th 5652/90. Based on lectures given at the 3rd Hellenic Summer School, 1989, published in the proceedings.

[5] L. Dixon, J. Harvey, C. Vafa, and E. Witten, Nucl. Phys. B 261 (1985) 678.

[6] L. Dixon, J. Harvey, C. Vafa, and E. Witten, Nucl. Phys. B 274 (1986) 285.

[7] K. Narain, M. Sarmadi and C. Vafa, Nucl. Phys. B 288 (1987) 551.

[8] K. Narain, Phys. Lett. 169 b (1986) 41.

[9] K. Narain, M. Sarmadi and E. Witten, Nucl. Phys. B 279 (1987) 369.

[10] L. E. Ibanez, H. P. Nilles and F. Quevedo, Phys. Lett. 187 B (1987) 25.

[11] L. E. Ibanez, J. E. Kim, H. P. Nilles and F. Quevedo, Phys. Lett. 191 B (1987) 3.

[12] A. Giveon, E. Rabinovici, and G. Veneziano, Nucl. Phys. B 322 (1989) 167.

[13] J. Lauer, J. Mas and H. P. Nilles, Nucl. Phys. B 351 (1991) 353.

[14] S. Ferrara, D. Lüst, A. Shapere and S. Theisen, Phys. Lett. 225 B (1989) 363.

[15] L. E. Ibanez and D. Lüst, em Nucl. Phys. B382 (1992) 305. 
[16] P. Ginsparg, Phys. Rev. D 38 (1987) 648.

[17] T. Mohaupt, Int. J. Mod. Phys. A8 (1993) 3529.

[18] P. Ginsparg, Applied Conformal Field Theory. In: E. Brezin, J. Zinn-Justin (ed.), Fields, Strings, Critical Phenomena, (Les Houches Summer School on Theoretical Physics 1988). North Holland (1990).

[19] A. Giveon, preprint RI-153-93, hep-th/9310016.

[20] L. E. Ibanez, J. Mas, H. P. Nilles and F. Quevedo, Nucl.Phys. B 301 (1988) 157.

[21] J. Erler, D. Jungnickel, J. Lauer, Phys. Rev D 45 (1992) 3651.

[22] J. Erler, A. Klemm, Comment on the Generation Number in Orbifold Compactifications. $M P I-P h-92-60, H E P-T h-9207111$.

[23] L. E. Ibanez, H. P. Nilles and F. Quevedo, Phys. Lett. B187 (1987) 25.

[24] J. F. Cornwell, Group Theory in Physics. Academic Press (1984), 3 vols.

[25] T. J. Hollowood, R. G. Myhill, Int. Jour. Mod. Phys. A 3 (1988) 899.

[26] V. G. Kac, Infinite dimensional Lie algebras. Cambridge University Press (1985).

[27] R. N. Cahn, Semi Simple Lie Algebras and Their Representations. Benjamin and Cummings (1984). 